\title{
Combinatorial study of Dellac configurations and $q$-extended normalized median Genocchi numbers
}

\author{
Ange Bigeni \\ Institut Camille Jordan \\ Universit Claude Bernard Lyon 1 \\ Lyon, France \\ bigeni@math.univ-lyon1.fr
}

Submitted: Feb 2, 2014; Accepted: May 3, 2014; Published: May 13, 2014

Mathematics Subject Classifications: 05C88, 05C89

\begin{abstract}
In two recent papers, Feigin proved that the Poincaré polynomials of the degenerate flag varieties have a combinatorial interpretation through Dellac configurations, and related them to the $q$-extended normalized median Genocchi numbers $\bar{c}_{n}(q)$ introduced by Han and Zeng, mainly by geometric considerations. In this paper, we give combinatorial proofs of these results by constructing statistic-preserving bijections between Dellac configurations and two other combinatorial models of $\bar{c}_{n}(q)$.
\end{abstract}

Keywords: Genocchi numbers; Dumont permutations; Dellac configurations; Dellac histories

\section{Introduction}

The Genocchi numbers $\left(G_{2 n}\right)_{n \geqslant 1}=(1,1,3,17, \ldots)[13]$ and the median Genocchi numbers $\left(H_{2 n+1}\right)_{n \geqslant 0}=(1,2,8,56, \ldots)$ [14] can be defined (see [5]) as the positive integers $G_{2 n}=$ $g_{2 n-1, n}$ and $H_{2 n+1}=g_{2 n+2,1}$ in the Seidel triangle $\left(g_{i, j}\right)_{1 \leqslant j \leqslant i}$ (see Figure 1) defined by

$$
\begin{aligned}
g_{2 p-1, j} & =g_{2 p-1, j-1}+g_{2 p, j}, \\
g_{2 p, j} & =g_{2 p-1, j}+g_{2 p, j+1},
\end{aligned}
$$

with $g_{1,1}=1$ and $g_{i, j}=0$ whenever $i<j$. 


\begin{tabular}{|c|c|c|c|c|c|c|c|}
\hline$i \backslash j$ & 1 & & 2 & & 3 & & 4 \\
\hline \multirow[t]{2}{*}{1} & 1 & & & & & & \\
\hline & $\downarrow$ & & & & & & \\
\hline \multirow[t]{2}{*}{2} & 1 & & & & & & \\
\hline & $\downarrow$ & & & & & & \\
\hline \multirow[t]{2}{*}{3} & 1 & $\rightarrow$ & 1 & & & & \\
\hline & $\downarrow$ & & $\downarrow$ & & & & \\
\hline \multirow[t]{2}{*}{4} & 2 & $\leftarrow$ & 1 & & & & \\
\hline & $\downarrow$ & & $\downarrow$ & & & & \\
\hline \multirow[t]{2}{*}{5} & 2 & $\rightarrow$ & 3 & $\rightarrow$ & 3 & & \\
\hline & $\downarrow$ & & $\downarrow$ & & $\downarrow$ & & \\
\hline \multirow[t]{2}{*}{6} & 8 & $\leftarrow$ & 6 & $\leftarrow$ & 3 & & \\
\hline & $\downarrow$ & & $\downarrow$ & & $\downarrow$ & & \\
\hline \multirow[t]{2}{*}{7} & 8 & $\rightarrow$ & 14 & $\rightarrow$ & 17 & $\rightarrow$ & 17 \\
\hline & $\downarrow$ & & $\downarrow$ & & $\downarrow$ & & $\downarrow$ \\
\hline \multirow[t]{3}{*}{8} & 56 & $\leftarrow$ & 48 & $\leftarrow$ & 34 & $\leftarrow$ & 17 \\
\hline & $\downarrow$ & & $\downarrow$ & & $\downarrow$ & & $\downarrow$ \\
\hline & $\vdots$ & & $\vdots$ & & $\vdots$ & & $\vdots$ \\
\hline
\end{tabular}

Figure 1: Seidel generation of the Genocchi numbers.

It is well known that $H_{2 n+1}$ is divisible by $2^{n}$ (see [1]) for all $n \geqslant 0$. The normalized median Genocchi numbers $\left(h_{n}\right)_{n \geqslant 0}=(1,1,2,7, \ldots)$ [15] are the positive integers defined by

$$
h_{n}=H_{2 n+1} / 2^{n} .
$$

Dumont [4] gave several combinatorial models of the Genocchi numbers and the median Genocchi numbers, among which are the Dumont permutations. We denote by $\mathfrak{S}_{n}$ the set of permutations of the set $[n]:=\{1,2, \ldots, n\}$, and by $\operatorname{inv}(\sigma)$ the number of inversions of a permutation $\sigma \in \mathfrak{S}_{n}$, i.e., the number of pairs $(i, j) \in[n]^{2}$ with $i<j$ and $\sigma(i)>\sigma(j)$. Broadly speaking, the number of inversions $\operatorname{inv}(w)$ of a word $w=l_{1} l_{2} \ldots l_{n}$ with $n$ letters in the alphabet $\mathbb{N}$ is the number of pairs $(i, j) \in[n]^{2}$ such that $i<j$ and $l_{i}>l_{j}$. In particular, the number $\operatorname{inv}(\sigma)$ associated with a permutation $\sigma \in \mathfrak{S}_{n}$ is the number $\operatorname{inv}(w)$ associated with the word $w=\sigma(1) \sigma(2) \ldots \sigma(n)$.

Definition 1.1. A Dumont permutation of order $2 n$ is a permutation $\sigma \in \mathfrak{S}_{2 n}$ such that $\sigma(2 i)<2 i$ and $\sigma(2 i-1)>2 i-1$ for all $i$. We denote by $\mathcal{D}_{n}$ the set of these permutations.

It is well-known (see [4]) that $H_{2 n+1}=\left|\mathcal{D}_{n+1}\right|$ for all $n \geqslant 0$. In [9], Han and Zeng introduced the set $\mathcal{G}_{n}^{\prime \prime}$ of normalized Genocchi permutations, which consists of permutations $\sigma \in \mathcal{D}_{n}$ such that for all $j \in[n-1]$, the two integers $\sigma^{-1}(2 j)$ and $\sigma^{-1}(2 j+1)$ have the same parity if and only if $\sigma^{-1}(2 j)<\sigma^{-1}(2 j+1)$, and they proved that $h_{n}=\left|\mathcal{G}_{n+1}^{\prime \prime}\right|$ for all $n \geqslant 0$. The number $h_{n}$ also counts the Dellac configurations of size $n$ (see [6]). 
Definition 1.2. A Dellac configuration of size $n$ is a tableau of width $n$ and height $2 n$ which contains $2 n$ dots between the lines $y=x$ and $y=n+x$, such that each row contains exactly one dot and each column contains exactly two dots. Let $D C(n)$ be the set of Dellac configurations of size $n$. An inversion of $C \in D C(n)$ is a pair $\left(d_{1}, d_{2}\right)$ of dots whose Cartesian coordinates in $C$ are respectively $\left(j_{1}, i_{1}\right)$ and $\left(j_{2}, i_{2}\right)$ such that $j_{1}<j_{2}$ and $i_{1}>i_{2}$. We denote by $\operatorname{inv}(C)$ the number of inversions of $C$. For example, the tableau depicted in Figure 2 is a Dellac configuration $C \in D C(3)$ with $\operatorname{inv}(C)=2$ inversions (represented by two segments).

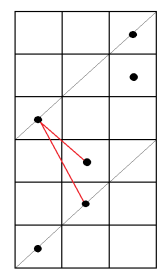

Figure 2: Dellac configuration $C \in D C(3)$ with $\operatorname{inv}(C)=2$ inversions.

In $[9,10]$, Han and Zeng defined the $q$-Gandhi polynomials of the second kind, denoted $\left(C_{n}(x, q)\right)_{n \geqslant 1}$, by $C_{1}(x, q)=1$ and $C_{n+1}(x, q)=(1+q x) \Delta_{q}\left(x C_{n}(x, q)\right)$, where

$$
\Delta_{q} P(x)=(P(1+q x)-P(x)) /(1+q x-x)
$$

for all polynomial $P(x)$. They proved that the polynomials $C_{n}(1, q)$ are $q$-analogs of the median Genocchi numbers $\left(C_{n}(1,1)=H_{2 n-1}\right)$. Furthermore, they gave a combinatorial interpretation of $C_{n}(1, q)$ through $\mathcal{D}_{n}$.

Theorem 1.1 (Han and Zeng, 1997). Let $n \geqslant 1$. For all $\sigma \in \mathcal{D}_{n}$, we define $\operatorname{st}(\sigma)$ as the number

$$
\operatorname{st}(\sigma)=n^{2}-\sum_{i=1}^{n} \sigma(2 i)-i n v\left(\sigma^{o}\right)-i n v\left(\sigma^{e}\right)
$$

where $\sigma^{o}$ and $\sigma^{e}$ are the two words $\sigma(1) \sigma(3) \ldots \sigma(2 n-1)$ and $\sigma(2) \sigma(4) \ldots \sigma(2 n)$ respectively. Then, the polynomial $C_{n}(1, q)$ has the following combinatorial interpretation:

$$
C_{n}(1, q)=\sum_{\sigma \in \mathcal{D}_{n}} q^{s t(\sigma)}
$$

By introducing the subset $\mathcal{G}_{n}^{\prime \prime} \subset \mathcal{D}_{n}$ of normalized Genocchi permutations and using the combinatorial interpretation provided by Theorem 1.1, Han and Zeng proved combinatorially that the polynomial $(1+q)^{n-1}$ divides $C_{n}(1, q)$, which gives birth to polynomials $\left(\bar{c}_{n}(q)\right)_{n \geqslant 1}$ defined by

$$
\bar{c}_{n}(q)=C_{n}(1, q) /(1+q)^{n-1} .
$$

This divisibility had previously been proved in the same paper with a continued fraction approach, as a corollary of the following theorem and a well-known result on continued fractions (see [8]). 
Theorem 1.2 (Han and Zeng, 1997). The generating function of the sequence $\left(\bar{c}_{n+1}(q)\right)_{n \geqslant 0}$ is

$$
\sum_{n \geqslant 0} \bar{c}_{n+1}(q) t^{n}=\frac{1}{1-\frac{\lambda_{1} t}{1-\frac{\lambda_{2} t}{1-\frac{\lambda_{3} t}{\ddots}}}}
$$

where $\lambda_{2 p-1}$ is the $q$-binomial coefficient $\left\{\begin{array}{c}p+1 \\ 2\end{array}\right\}_{q}:=\left(1-q^{p+1}\right)\left(1-q^{p}\right) /\left(1-q^{2}\right)(1-q)$ and $\lambda_{2 p}=q \lambda_{2 p-1}$ for all $p \geqslant 1$.

The polynomials $\left(\bar{c}_{n}(q)\right)_{n \geqslant 1}$ are $q$-refinements of normalized median Genocchi numbers: $\bar{c}_{n}(1)=h_{n-1}$ for all $n \geqslant 1$. They are named $q$-extended normalized median Genocchi numbers. In $\S 2.1$, we give a combinatorial interpretation of $\bar{c}_{n}(q)$ by slightly adjusting the definition of normalized Genocchi permutations. In [6, 7], Feigin introduced a $q$-analog of the normalized median Genocchi number $h_{n}$ with the Poincaré polynomial $P_{\mathcal{F}_{n}^{a}}(q)$ of the degenate flag variety $\mathcal{F}_{n}^{a}$ (whose Euler characteristic is $P_{\mathcal{F}_{n}^{a}}(1)=h_{n}$ ), and gave a combinatorial interpretation of $P_{\mathcal{F}_{n}^{a}}(q)$ through Dellac configurations.

Theorem 1.3 (Feigin, 2012). For all $n \geqslant 0$, the polynomial $P_{\mathcal{F}_{n}^{a}}(q)$ is generated by $D C(n)$ :

$$
P_{\mathcal{F}_{n}^{a}}(q)=\sum_{C \in D C(n)} q^{2 i n v(C)}
$$

The degree of the polynomial $P_{\mathcal{F}_{n}^{a}}(q)$ being $n(n+1)$ (for algebraic considerations, or because every Dellac configuration $C \in D C(n)$ has at most $\left(\begin{array}{l}n \\ 2\end{array}\right)$ inversions, see $\left.\S 2.1\right)$, Feigin introduced the following $q$-analog of $h_{n}$ :

$$
\tilde{h}_{n}(q)=q^{\left(\begin{array}{c}
n \\
2
\end{array}\right)} P_{\mathcal{F}_{n}^{a}}\left(q^{-1 / 2}\right)=\sum_{C \in D C(n)} q^{\left(\begin{array}{c}
n \\
2
\end{array}\right)-\operatorname{inv}(C)},
$$

and proved the following theorem by using the geometry of quiver Grassmannians (see [11]) and Flajolet's theory of continued fractions [8].

Theorem 1.4 (Feigin, 2012). The generating function $\sum_{n \geqslant 0} \tilde{h}_{n}(q) t^{n}$ has the continued fraction expansion of Formula (4).

Corollary 1.5 (Feigin, 2012). For all $n \geqslant 0$, we have $\tilde{h}_{n}(q)=\bar{c}_{n+1}(q)$.

This raises two questions.

1. Prove combinatorially Corollary 1.5 by constructing a bijection between Dellac configurations and some appropriate model of $\bar{c}_{n}(q)$ which preserves the statistics.

2. Prove combinatorially Theorem 1.4 within the framework of Flajolet's theory of continued fractions by defining a combinatorial model of $\tilde{h}_{n}(q)$ related to Dyck paths (see [8]), and constructing a statistic-preserving bijection between Dellac configurations and that new model. 
The aim of this paper is to answer above two questions. We answer the first one in $\S 2$. In $\S 2.1$, we define a combinatorial model of $\bar{c}_{n}(q)$ through normalized Dumont permutations, and we provide general results about Dellac configurations. In $\S 2.2$, we enounce and prove Theorem 2.2, which connects Dellac configurations to normalized Dumont permutations through a stastistic-preserving bijection, and implies immediatly Corollary 1.5. We answer the second question in $\S 3$. In $\S 3.1$, we recall the definition of a Dyck path and some results of Flajolet's theory of continued fractions. In $\S 3.2$, we define Dellac histories, which consist of Dyck paths weighted with pairs of integers, and we show that their generating function has the continued fraction expansion of Formula (4). In §3.3, we enounce and prove Theorem 3.3, which connects Dellac configurations to Dellac histories through a statistic-preserving bijection, thence proving Theorem 1.4 combinatorially.

\section{Connection between Dellac configurations and Du- mont permutations}

In $\S 2.1$, we define normalized Dumont permutations of order $2 n$, whose set is denoted by $\mathcal{D}_{n}^{\prime}$, and we prove that they generate $\bar{c}_{n}(q)$ with respect to the statistic st defined in Formula (1), then we define the label of a Dellac configuration and a switching transformation on the set $D C(n)$. In $\S 2.2$, we enounce Theorem 2.2 and we prove it. To do so, we first give two maps $\phi: D C(n) \rightarrow \mathcal{D}_{n+1}$ and $\varphi: \mathcal{D}_{n+1} \rightarrow D C(n)$, and we prove that $\phi$ and $\varphi_{\mid \mathcal{D}_{n+1}^{\prime}}$ are inverse maps. Then, we show that Equation (6) is true for all $C \in D C(n)$, by showing that it is true for some particular $C^{0} \in D C(n)$, then by connecting $C^{0}$ to every other $C \in D C(n)$ thanks to the switching transformation, which happens to preserve Equation (6).

\subsection{Preliminaries}

\subsubsection{Combinatorial interpretation of $\bar{c}_{n}(q)$.}

Definition 2.1. A normalized Dumont permutation of order $2 n$ is a permutation $\sigma \in \mathcal{D}_{n}$ such that, for all $j \in[n-1]$, the two integers $\sigma^{-1}(2 j)$ and $\sigma^{-1}(2 j+1)$ have the same parity if and only if $\sigma^{-1}(2 j)>\sigma^{-1}(2 j+1)$. Let $\mathcal{D}_{n}^{\prime} \subset \mathcal{D}_{n}$ be the set of these permutations.

Proposition 2.1. For all $n \geqslant 1$, we have $\bar{c}_{n}(q)=\sum_{\sigma \in \mathcal{D}_{n}^{\prime}} q^{s t(\sigma)}$.

Proof. Let $j \in[n-1]$ and $\sigma \in \mathcal{D}_{n}$. Recall that $s t(\sigma)=n^{2}-\sum_{i=1}^{n} \sigma(2 i)-\operatorname{inv}\left(\sigma^{o}\right)-\operatorname{inv}\left(\sigma^{e}\right)$. It is easy to see that the composition $\sigma^{\prime}=(2 j, 2 j+1) \circ \sigma$ of $\sigma$ with the transposition $(2 j, 2 j+1)$ is still a Dumont permutation, and that if $\sigma$ fits the condition $C(j)$ defined as

$$
\sigma^{-1}(2 j)>\sigma^{-1}(2 j+1) \Leftrightarrow \sigma^{-1}(2 j) \text { and } \sigma^{-1}(2 j+1) \text { have the same parity, }
$$

then $\operatorname{st}\left(\sigma^{\prime}\right)=\operatorname{st}(\sigma)+1$. Now, if we denote by $\mathcal{D}_{n}^{j} \subset \mathcal{D}_{n}$ the subset of permutations that fit the condition $C(j)$, then $\mathcal{D}_{n}$ is the disjoint union $\mathcal{D}_{n}^{j} \sqcup\left((2 j, 2 j+1) \circ \mathcal{D}_{n}^{j}\right)$, where 
$(2 j, 2 j+1) \circ \mathcal{D}_{n}^{j}$ is the set $\left\{(2 j, 2 j+1) \circ \sigma, \sigma \in \mathcal{D}_{n}^{j}\right\}$. Since $\operatorname{st}((2 j, 2 j+1) \circ \sigma)=\operatorname{st}(\sigma)+1$ for all $\sigma \in \mathcal{D}_{n}^{j}$, Formula (2) of Theorem 1.1 becomes

$$
C_{n}(1, q)=(1+q) \sum_{\sigma \in \mathcal{D}_{n}^{j}} q^{s t(\sigma)}
$$

This yields immediatly:

$$
C_{n}(1, q)=(1+q)^{n-1} \sum_{\sigma \in \bigcap_{j=1}^{n-1} \mathcal{D}_{n}^{j}} q^{s t(\sigma)}=(1+q)^{n-1} \sum_{\sigma \in \mathcal{D}_{n}^{\prime}} q^{s t(\sigma)} .
$$

The proposition then follows from Formula (3).

\subsubsection{Label of a Dellac configuration}

Definition 2.2. Let $C \in D C(n)$. For all $i \in[n]$, the dot of the $i$-th line of $C$ (from bottom to top) is labeled by the integer $e_{i}=2 i+2$, and the dot of the $(n+i)$-th line is labeled by the integer $e_{n+i}=2 i-1$ (see Figure 3 for an example).

From now on, we will assimilate each dot of a Dellac configuration into its label.

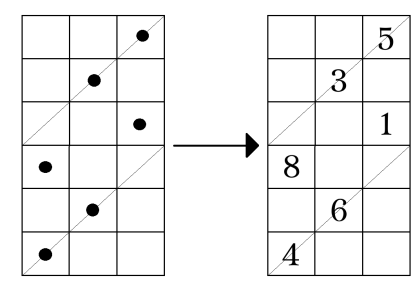

Figure 3: Label of a Dellac configuration $C \in D C(3)$.

Definition 2.3 (Particular dots). Let $C \in D C(n)$. For all $j \in[n]$, we define $i_{1}^{C}(j)<i_{2}^{C}(j)$ such that the two dots of the $j$-th column of $C$ (from left to right) are $e_{i_{1}^{C}(j)}$ and $e_{i_{2}^{C}(j)}$. When there is no ambiguity, we write $e_{i_{1}(j)}$ and $e_{i_{2}(j)}$ instead of $e_{i_{1}^{C}(j)}$ and $e_{i_{2}^{C}(j)}$.

Finally, for all $i \in[n]$, we define the integers $p_{C}(i)$ and $q_{C}(i)$ such that $e_{p_{C}(i)}$ and $e_{n+q_{C}(i)}$ are respectively the $i$-th even dot and $i$-th odd dot of the sequence

$$
\left(e_{i_{1}(1)}, e_{i_{2}(1)}, e_{i_{1}(2)}, e_{i_{2}(2)}, \ldots, e_{i_{1}(n)}, e_{i_{2}(n)}\right) .
$$

For example, in Figure 3, we have $\left(e_{i_{1}(2)}, e_{i_{2}(2)}\right)=(6,3)=\left(e_{2}, e_{5}\right)=\left(e_{p_{C}(3)}, e_{3+q_{C}(1)}\right)$.

Remark 2.1. For all $i \in[2 n]$, if the $\operatorname{dot} e_{i}$ appears in the $j_{i}$-th column of $C$, then, by Definition 1.2 , we have $j_{i} \leqslant i \leqslant j_{i}+n$. As a result, the first $j$ columns of $C$ always contain the $j$ even dots

$$
e_{1}, e_{2}, \ldots, e_{j}
$$


and the only odd dots they may contain are

$$
e_{n+1}, e_{n+2}, \ldots, e_{n+j} \text {. }
$$

Likewise, the last $n-j+1$ columns of $C$ always contain the $n-j+1$ odd dots

$$
e_{n+j}, e_{n+j+1}, \ldots, e_{2 n}
$$

and the only even dots they may contain are

$$
e_{j}, e_{j+1}, e_{j+2}, \ldots, e_{n} .
$$

Remark 2.2. Let $C \in D C(n)$ and $j \in[n]$. If the $j$-th column of $C$ contains the even dot $e_{i \leqslant n}=2 i+2$, then, since $j \leqslant i$, we have $e_{i} \in\{2 j+2,2 j+4, \ldots, 2 n+2\}$. Similarly, if the $j$-th column of $C$ contains the odd dot $e_{i>n}=2(i-n)-1$, since $i \leqslant j+n$, we have $e_{i} \in\{1,3, \ldots, 2 j-1\}$. As a result, we obtain the following equivalences:

$$
e_{i_{1}^{C}(j)}>e_{i_{2}^{C}(j)} \Leftrightarrow i_{1}^{C}(j) \leqslant n<i_{2}^{C}(j) \Leftrightarrow e_{i_{1}^{C}(j)} \text { and } e_{i_{2}^{C}(j)} \text { have different parities. }
$$

Definition 2.4 (Particular configurations). For all $n \geqslant 1$, we denote by $C_{0}(n)$ (respectively $\left.C_{1}(n)\right)$ the Dellac configuration of size $n$ such that $\left(e_{i_{1}(j)}, e_{i_{2}(j)}\right)=\left(e_{2 j-1}, e_{2 j}\right)$ (resp. $\left.\left(e_{i_{1}(j)}, e_{i_{2}(j)}\right)=\left(e_{j}, e_{n+j}\right)\right)$ for all $j \in[n]$. For example $C_{0}(3)$ (on the left) and $C_{1}(3)$ (on the right) are the two configurations depicted in Figure 4.

It is obvious that $C_{0}(n)$ is the unique Dellac configuration of size $n$ with 0 inversion, and that $\operatorname{inv}\left(C_{1}(n)\right)=\left(\begin{array}{l}n \\ 2\end{array}\right)$. We can also prove by induction on $n \geqslant 1$ that every Dellac configuration $C \in D C(n)$ has at most $\left(\begin{array}{l}n \\ 2\end{array}\right)$ inversions with equality if and only if $C=C_{1}(n)$.
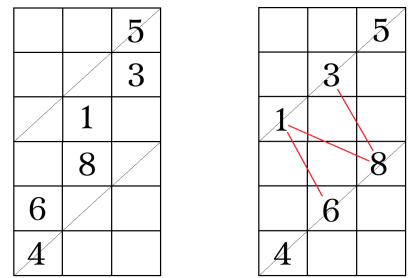

Figure 4: $C_{0}(3)$ and $C_{1}(3)$.

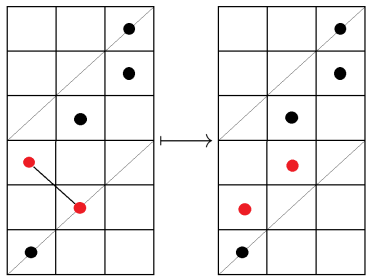

Figure 5: $C \in D C(3) \mapsto S w^{2}(C) \in D C(3)$.

\subsubsection{Refinements of the inv statistic on $D C(n)$}

Definition 2.5. Let $C \in D C(n)$ and $i \in[2 n]$. We define the number $l_{C}\left(e_{i}\right)$ (resp. $\left.r_{C}\left(e_{i}\right)\right)$ as the number of inversions of $C$ between the $\operatorname{dot} e_{i}$ and any $\operatorname{dot} e_{i^{\prime}}$ with $i^{\prime}>i$ (resp. $i^{\prime}<i$ ). For example, if $C=C_{1}(3)$ (see Figure 4), then $l_{C}(6)=r_{C}(3)=1$ and $r_{C}(1)=l_{C}(8)=2$. 


\subsubsection{Switching of a Dellac configuration}

In the following definition, we provide a tool which transforms a Dellac configuration of $D C(n)$ into a slightly modified tableau, which is not necessarily a Dellac configuration and consequently brings the notion of switchability.

Definition 2.6. Let $C \in D C(n)$ and $i \in[2 n-1]$. We denote by $S w^{i}(C)$ the tableau obtained by switching the two consecutive dots $e_{i}$ and $e_{i+1}$ (i.e., inserting $e_{i}$ in $e_{i+1}$ 's column and $e_{i+1}$ in $e_{i}$ 's column). If the tableau $S w^{i}(C)$ is still a Dellac configuration, we say that $C$ is switchable at $i$. In Figure 5 , we give an example $C \in D C(3)$ switchable at 2 .

It is easy to verify the following assertions.

Fact 2.1. If $C \in D C(n)$ is switchable at $i$, then $\left|\operatorname{inv}\left(\left(S w^{i}(C)\right)\right)-i n v(C)\right| \leqslant 1$.

Fact 2.2. A Dellac configuration $C \in D C(n)$ is switchable at $i \in[2 n-1]$ if and only if $C$ and $i$ satisfy one of the two following conditions:

(1) $i \leqslant n$ and if $e_{i+1}$ is in the $j_{i+1}$-th column of $C$, then $j_{i+1}<i+1$.

(2) $i>n$ and if $e_{i}$ is in the $j_{i}$-th column of $C$, then $j_{i}>i-n$.

In particular :

Fact 2.3. If $C$ is switchable at $i$, then $S w^{i}(C)$ is still switchable at $i$ and $S w^{i}\left(S w^{i}(C)\right)=$ $C$.

Fact 2.4. If $e_{i}$ and $e_{i+1}$ are in the same column of $C$, then $C$ is switchable at $i$ and $C=S w^{i}(C)$.

Fact 2.5. If $\left(e_{i}, e_{i+1}\right)$ is an inversion of $C$, then $C$ is switchable at $i$ and $\operatorname{inv}\left(S w^{i}(C)\right)=$ inv $(C)-1$ (like in Figure 5).

Fact 2.6. A Dellac configuration $C \in D C(n)$ is always switchable at $n$.

\subsection{Construction of a statistic-preserving bijection}

In this part, we intend to prove the following result.

Theorem 2.2. There exists a bijection $\phi: D C(n) \rightarrow \mathcal{D}_{n+1}^{\prime}$ such that the equality

$$
\operatorname{st}(\phi(C))=\left(\begin{array}{l}
n \\
2
\end{array}\right)-i n v(C)
$$

is true for all $C \in D C(n)$.

In the following, we define $\phi: D C(n) \rightarrow \mathcal{D}_{n+1}^{\prime}$ and in order to prove that it is bijective, we construct $\varphi: \mathcal{D}_{n+1} \rightarrow D C(n)$ such that $\phi$ and $\varphi_{\mid \mathcal{D}_{n+1}^{\prime}}$ are inverse maps. 


\subsubsection{Bijections}

Definition of $\phi$. We define $\phi: D C(n) \rightarrow \mathfrak{S}_{2 n+2}$ by mapping $C \in D C(n)$ to the permutation $\phi(C) \in \mathfrak{S}_{2 n+2}$ defined by

$$
\phi(C)^{-1}=2 e_{i_{2}(1)} e_{i_{1}(1)} e_{i_{2}(2)} e_{i_{1}(2)} \ldots e_{i_{2}(n)} e_{i_{1}(n)}(2 n+1)
$$

where we recall that $e_{i_{1}(j)}$ and $e_{i_{2}(j)}$ are respectively the lower and upper dots of the $j$-th column of $C$ for all $j \in[n]$. In other words, the permutation $\phi(C)$ is defined by $(\phi(C)(2), \phi(C)(2 n+1))=(1,2 n+2)$ and $\left(\phi(C)\left(e_{i_{1}(j)}\right), \phi(C)\left(e_{i_{2}(j)}\right)\right)=(2 j+1,2 j)$ for all $j \in[n]$.

Example 2.1. If $C \in D C(3)$ is the Dellac configuration depicted in Figure 6, we obtain $\phi(C)^{-1}=28416537$.

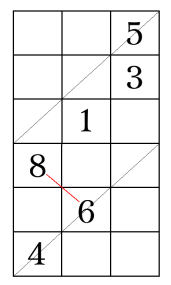

Figure 6: $C \in D C(3)$.

Proposition 2.3. For all $C \in D C(n)$, the permutation $\phi(C)$ is a normalized Dumont permutation.

Proof. Let $\sigma$ be $\phi(C)$. It is a Dumont permutation : $(\sigma(2), \sigma(2 n+1))=(1,2 n+2)$ and for all $i \in\{2,3, \ldots, n-1\}$, if the $\operatorname{dot} 2 i=e_{i-1}$ is in the $j$-th column of $C$ (resp. if the dot $2 i+1=e_{n+1+i}$ is in the $j^{\prime}$-th column of $\left.C\right)$, then $\sigma(2 i)=\sigma\left(e_{i-1}\right) \leqslant 2 j+1<2 i$ because $j \leqslant i-1$ (resp. $\sigma(2 i+1)=\sigma\left(e_{n+1+i}\right) \geqslant 2 j^{\prime}>2 i+1$ because $\left.n+1+i \leqslant j^{\prime}+n\right)$. It is also normalized according to Remark 2.2.

Definition of $\boldsymbol{\varphi}$. Let $\mathcal{T}_{n}$ be the set of tableaux of size $n \times 2 n$ whose each row contains one dot and each column contains two dots. We define $\varphi: \mathcal{D}_{n+1} \rightarrow \mathcal{T}_{n}$ by mapping $\sigma \in \mathcal{D}_{n+1}$ to the tableau $\varphi(\sigma) \in \mathcal{T}_{n}$ whose $j$-th column contains the two dots labelled by $\sigma^{-1}(2 j)$ and $\sigma^{-1}(2 j+1)$ for all $j \in[n]$.

Proposition 2.4. For all $\sigma \in \mathcal{D}_{n+1}$, the tableau $\varphi(\sigma)$ is a Dellac configuration.

Proof. Let $j \in[n]$ and $i \in[2 n]$ such that $\varphi(\sigma)$ contains a dot in the box $(j, i)$ (i.e., the $j$-th column of $\varphi(\sigma)$ contains the dot $\left.e_{i}\right)$. By definition $2 j \leqslant \sigma\left(e_{i}\right) \leqslant 2 j+1$. If $i \leqslant n$, then $e_{i}=2 i+2$ and $2 j \leqslant \sigma(2 i+2)<2 i+2$ thence $j \leqslant i<j+n$. Else $e_{i}=2(i-n)-1$ and $2 j+1 \geqslant \sigma(2(i-n)-1)>2(i-n)-1$ thence $j \geqslant i-n>0 \geqslant j-n$. In either case we obtain $j \leqslant i \leqslant j+n$ so $\varphi(\sigma) \in D C(n)$. 
Example 2.2. Consider the permutation $\sigma=41726583 \in \mathcal{D}_{4}$. From $\sigma^{-1}=24816537$, we obtain the Dellac configuration $\varphi(\sigma)$ illustrated in Figure 6.

It is easy to verify that $\phi \circ \varphi_{\mid \mathcal{D}_{n+1}^{\prime}}=I d_{\mathcal{D}_{n+1}^{\prime}}$ and $\varphi \circ \phi=I d_{D C(n)}$.

Remark 2.3. There is a natural interpretation in terms of group action : in the proof of Proposition 2.1, we show that the subgroup of $\mathfrak{S}_{2 n+2}$ generated by the $n$ permutations $(2,3),(4,5), \ldots,(2 n, 2 n+1)$, freely operates by left multiplication on $\mathcal{D}_{n+1}$, and that each orbit of that action contains exactly one normalized Dumont permutation. Also, the orbits are indexed by elements of $D C(n)$ : two permutations $\sigma_{1}$ and $\sigma_{2} \in \mathcal{D}_{n+1}$ are in the same orbit if and only if $\varphi\left(\sigma_{1}\right)=\varphi\left(\sigma_{2}\right)$, and for all $\sigma \in \mathcal{D}_{n+1}$, the permutation $\phi(\varphi(\sigma))$ is the unique normalized Dumont permutation in the orbit of $\sigma$.

Example 2.3. In Examples 2.1 and 2.2, we have $\varphi(\phi(C))=C$ and $\phi(\varphi(\sigma))=(2,3) \circ \sigma$.

\subsubsection{Alternative algorithm}

Definition 2.7. Let $\left(y_{1}, y_{2}, \ldots, y_{2 n}\right)$ be the sequence $(3,2,5,4, \ldots, 2 n+1,2 n)$. For all $C \in D C(n)$, we define the permutation $\tau_{C} \in \mathfrak{S}_{2 n}$ by $\phi(C)\left(e_{i}\right)=y_{\tau_{C}(i)}$ for all $i \in[2 n]$.

Lemma 2.5. Let $C \in D C(n)$ and $(p, q) \in[2 n]^{2}$ such that $p<q$. Then $\left(e_{p}, e_{q}\right)$ is an inversion of $C$ if and only if $(p, q)$ is an inversion of $\tau_{C}$, i.e., if $\tau_{C}(p)>\tau_{C}(q)$.

Proof. Recall that if the dot $e_{i}$ is located in the $j$-th column of $C$, then $\phi(C)\left(e_{i}\right)=2 j$ or $2 j+1$. Consequently, since $y_{i}=i$ if $i$ is even, and $y_{i}=i+2$ if $i$ is odd, then $\tau_{C}(i)=2 j$ or $2 j-1$. Now let $1 \leqslant p<q \leqslant 2 n$, and let $\left(j_{p}, j_{q}\right)$ such that the dot $e_{p}$ (resp. $\left.e_{q}\right)$ is located in the $j_{p}$-th column (resp. $j_{q}$-th column) of $C$. If $\left(e_{p}, e_{q}\right)$ is an inversion of $C$, i.e., if $j_{p}>j_{q}$, then $\tau_{C}(p) \geqslant 2 j_{p}-1>2 j_{q} \geqslant \tau_{C}(q)$ and $(p, q)$ is an inversion of $\tau_{C}$. Reciprocally, if $\tau_{C}(p)>\tau_{C}(q)$, then $2 j_{p} \geqslant \tau_{C}(p)>\tau_{C}(q) \geqslant 2 j_{q}-1$, hence $j_{p} \geqslant j_{q}$. Now suppose that $j_{p}=j_{q}=: j$. It means that $e_{p}$ and $e_{q}$ are the lower dot and the upper dot of the $j$-th column respectively, which translates into $y_{\tau_{C}(p)}=\phi(C)\left(e_{p}\right)=2 j+1$ and $y_{\tau_{C}(q)}=\phi(C)\left(e_{q}\right)=2 j$. Consequently, we obtain $\tau_{C}(p)=2 j-1$ and $\tau_{C}(q)=2 j$, which is in contradiction with $\tau_{C}(p)>\tau_{C}(q)$. So $j_{p}>j_{q}$ and $\left(e_{p}, e_{q}\right)$ is an inversion of $C$.

Proposition 2.6 (Alternative algorithm for the map $\phi: \boldsymbol{D C}(\boldsymbol{n}) \rightarrow \mathcal{D}_{\boldsymbol{n + 1}}^{\prime}$ ). Let $C \in$ $D C(n)$. For all $i \in[2 n]$, we have $\tau_{C}(i)=i+l_{C}\left(e_{i}\right)-r_{C}\left(e_{i}\right)$.

Example 2.4. Consider the following Dellac configuration $C \in D C(3)$.

\begin{tabular}{l|l|l|l|}
\cline { 2 - 4 }$y_{6}=6$ & & & 5 \\
$y_{5}=7$ & & & 3 \\
$y_{4}=4$ & 1 & & \\
$y_{3}=5$ & & 8 & \\
$y_{2}=2$ & & 6 & \\
$y_{1}=3$ & 4 & & \\
\hline
\end{tabular}

By Proposition 2.6, we obtain $\phi(C)=21736584$. This is coherent with the algorithm given in Definition 3.5, which says that $\phi(C)^{-1}=21486537$. 
Proof of Lemma 2.6. From Lemma 2.5, we know that

$$
\left\{\begin{array}{l}
l_{C}\left(e_{i}\right)=\left|\left\{k>i \mid \tau_{C}(k)<\tau_{C}(i)\right\}\right|, \\
r_{C}\left(e_{i}\right)=\left|\left\{k<i \mid \tau_{C}(k)>\tau_{C}(i)\right\}\right| .
\end{array}\right.
$$

So, the lemma follows from the well-known equality

$$
\pi(i)=i+|\{k>i \mid \pi(k)<\pi(i)\}|-|\{k<i \mid \pi(k)>\pi(i)\}|
$$

for all permutation $\pi \in \mathfrak{S}_{m}$ and for all integer $m \geqslant 1$.

\subsubsection{Switchability and Dumont permutations}

We have built a bijection $\phi: D C(n) \rightarrow \mathcal{D}_{n+1}^{\prime}$. To demonstrate Formula (6), we will use the notion of switchability defined in $\S 2.1$, by showing that if Formula (6) is true for some particular configuration $C^{0}$, and if $C^{1}$ is a configuration connected to $C^{0}$ by a switching transformation, then Formula (6) is also true for $C^{1}$. We will also need Lemma 2.7 and Proposition 2.8 to prove (in Proposition 2.9) that any two Dellac configurations are connected by a sequence of switching transformations.

Lemma 2.7. Let $\sigma \in \mathcal{D}_{n+1}$ and $i \in[2 n-1]$. We denote by $\sigma^{\prime}$ the composition $\sigma \circ\left(e_{i}, e_{i+1}\right)$ of the transposition $\left(e_{i}, e_{i+1}\right)$ with the permutation $\sigma$. The Dellac configuration $\varphi(\sigma)$ is switchable at $i$ if and only if $\sigma^{\prime}$ is still a Dumont permutation, and in that case $\varphi\left(\sigma^{\prime}\right)=$ $S w^{i}(\varphi(\sigma))$.

Proof. Let $T$ be the tableau $S w^{i}(\varphi(\sigma))$. If $T$ is a Dellac configuration, one can check that $\sigma^{\prime} \in \mathcal{D}_{n+1}$ thanks to Fact 2.2. Reciprocally, if $\sigma^{\prime}$ is a Dumont permutation, we may consider the Dellac configuration $\varphi\left(\sigma^{\prime}\right)$. For all $j \in[n]$, let $\left(e_{i_{1}(j)}, e_{i_{2}(j)}\right)$ (with $i_{1}(j)<$ $\left.i_{2}(j)\right)$ be the two dots of the $j$-th column of $\varphi(\sigma)$, and $\left(e_{i_{1}^{\prime}(j)}, e_{i_{2}^{\prime}(j)}\right)\left(\right.$ with $\left.i_{1}^{\prime}(j)<i_{2}^{\prime}(j)\right)$ the two dots of the $j$-th column of $\varphi\left(\sigma^{\prime}\right)$. Then $e_{i_{1}^{\prime}(j)}=\sigma^{\prime-1}(2 j+1)=\left(e_{i}, e_{i+1}\right) \circ \sigma^{-1}(2 j+1)=$ $\left(e_{i}, e_{i+1}\right)\left(e_{i_{1}(j)}\right)$ and $e_{i_{2}^{\prime}(j)}=\sigma^{-1}(2 j)=\left(e_{i}, e_{i+1}\right) \circ \sigma^{-1}(2 j)=\left(e_{i}, e_{i+1}\right)\left(e_{i_{2}(j)}\right)$ for all $j$, which exactly translates into $\varphi\left(\sigma^{\prime}\right)=S w^{i}(\varphi(\sigma))=T$.

The following result is easy.

Proposition 2.8. In the setting of Lemma 2.7, if $\varphi(\sigma)$ is switchable at $i$, then the following conditions are equivalent.

1. $\varphi\left(\sigma^{\prime}\right) \neq \varphi(\sigma)$;

2. the two dots $e_{i}$ and $e_{i+1}$ are not in the same column of $\varphi(\sigma)$;

3. $\operatorname{inv}\left(\varphi\left(\sigma^{\prime}\right)\right)-\operatorname{inv}(\varphi(\sigma))= \pm 1$;

4. $\phi(\varphi(\sigma)) \circ\left(e_{i}, e_{i+1}\right) \in \mathcal{D}_{n+1}^{\prime}$;

5. $\phi\left(\varphi\left(\sigma^{\prime}\right)\right)=\phi(\varphi(\sigma)) \circ\left(e_{i}, e_{i+1}\right)$. 
Proposition 2.9. Let $\left(C_{1}, C_{2}\right) \in D C(n)^{2}$. There exists a finite sequence of switching transformations from $C_{1}$ to $C_{2}$, i.e., a sequence $\left(C^{0}, C^{1}, \ldots, C^{m}\right)$ in $D C(n)$ for some $m \geqslant 0$ such that $\left(C^{0}, C^{m}\right)=\left(C_{1}, C_{2}\right)$ and such that $C^{k}=S w^{i_{k-1}}\left(C^{k-1}\right)$ for some index $i_{k-1} \in[2 n]$, for all $k \in[m]$.

Proof. From Fact 2.3, it is sufficient to prove that for all $C \in D C(n)$, there exists a finite sequence of switching transformations from $C$ to $C_{0}(n)$, the unique Dellac configuration of size $n$ with 0 inversion (see Definition 2.4). If $C=C_{0}(n)$, the statement is obvious. Else, let $C^{0}=C$. From Lemma 2.5, for all $i \in[2 n]$, the pair $\left(e_{i}, e_{i+1}\right)$ is an inversion of $C^{0}$ if and only if the integer $i$ is a descent of $\tau_{C^{0}}$, i.e., if $\tau_{C^{0}}(i)>\tau_{C^{0}}(i+1)$. Now, from Proposition 2.6, the permutation $\tau_{C_{0}(n)}$ is the identity map $I d$ of $\mathfrak{S}_{2 n+2}$. Consequently, since $C^{0} \neq C_{0}(n)$, we have $\tau_{C^{0}} \neq I d_{\mathfrak{S}_{2 n}}$, so $\tau_{C^{0}}$ has at least one descent. Let $i_{0}$ be one of those descents, and let $C^{1}=S w^{i_{0}}\left(C^{0}\right) \in D C(n)$. Since $\left(e_{i_{0}}, e_{i_{0}+1}\right)$ is an inversion of $C^{0}$, in particular $e_{i_{0}}$ and $e_{i_{0}+1}$ are not in the same column, so, from Proposition 2.8, we have $\phi\left(C^{1}\right)=\phi\left(C^{0}\right) \circ\left(e_{i_{0}}, e_{i_{0}+1}\right)$, hence $\tau_{C^{1}}=\tau_{C^{0}} \circ\left(i_{0}, i_{0}+1\right)$. Consequently, since $i_{0}$ is a descent of $\tau_{C^{0}}$, it is not a descent of $\tau_{C^{1}}$. Iterating the process with $C^{1}$, and by induction, we build a finite sequence of switching transformations $\left(C^{0}, C^{1}, \ldots, C^{m}\right)$ such that $\tau_{C^{m}}$ has no descent, i.e., such that $\tau_{C^{m}}=I d=\tau_{C_{0}(n)}$, which implies $C^{m}=C_{0}(n)$.

Example 2.5. In Figure 7, we give a graph whose vertices are the $h_{3}=7$ elements of $D C(3)$, and in which two Dellac configurations are connected by an edge if they are connected by a switching transformation.

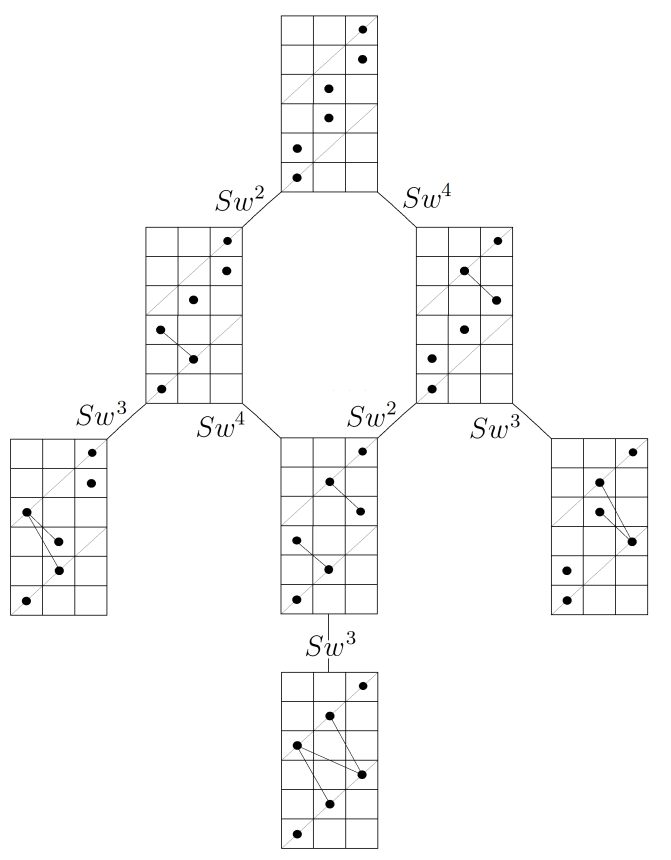

Figure 7: The switching transformations of $D C(3)$. 


\subsubsection{Proof of the statistic preservation formula (6)}

We are going to prove that Formula (6) is true for all $C \in D C(n)$, which will derive the proof of Theorem 2.2. First notice that it is true for $C=C_{1}(n)$, the unique Dellac configuration with $\left(\begin{array}{l}n \\ 2\end{array}\right)$ inversions (see Definition 2.4): indeed $\phi\left(C_{1}(n)\right)$ is the involution $214365 \ldots(2 n+2)(2 n+1)$, consequently the two permutations $\phi\left(C_{1}(n)\right)^{e}=135 \ldots(2 n+1)$ and $\phi\left(C_{1}(n)\right)^{\circ}=246 \ldots(2 n+2)$ have no inversion, hence

$$
s t\left(\phi\left(C_{1}(n)\right)\right)=(n+1)^{2}-(1+3+5+\ldots+(2 n+1))=0 .
$$

Let $C \in D C(n)$. From Lemma 2.9, there exists a finite sequence of switching transformations $\left(C^{0}, C^{1}, \ldots, C^{m}\right)$ from $C^{0}=C_{1}(n)$ to $C^{m}=C$. For all $k \in\{0,1, \ldots, m-1\}$, let $i_{k} \in[2 n]$ such that $C^{k+1}=S w^{i_{k}}\left(C^{k}\right)$. We can suppose that $C_{k+1} \neq C_{k}$, i.e., that $\operatorname{inv}\left(C^{k+1}\right)=\operatorname{inv}\left(C^{k}\right) \pm 1$. Since Formula (6) is true for $C_{1}(n)$, it will be true for $C$ by induction if we show that

$$
\operatorname{st}\left(\phi\left(C^{k+1}\right)\right)-\operatorname{st}\left(\phi\left(C^{k}\right)\right)=\operatorname{inv}\left(C^{k}\right)-\operatorname{inv}\left(C^{k+1}\right)
$$

for all $k$. We know that the number $\operatorname{inv}\left(C^{k}\right)-\operatorname{inv}\left(C^{k+1}\right)$ equals \pm 1 . From Fact 2.3, we have $S w^{i_{k}}\left(C^{k+1}\right)=C^{k}$. Then, provided that $C^{k}$ is replaced by $S w^{i_{k}}\left(C^{k}\right)=C^{k+1}$, we can assume that the number $\operatorname{inv}\left(C^{k}\right)-\operatorname{inv}\left(C^{k+1}\right)$ equals 1 , which means the pair $\left(e_{i_{k}}, e_{i_{k+1}}\right)$ is an inversion of $C^{k}$. Consequently, to achieve the proof of Theorem 2.2, it suffices to prove the equality

$$
\operatorname{st}\left(\phi\left(C^{k+1}\right)\right)-\operatorname{st}\left(\phi\left(C^{k}\right)\right)=1
$$

under the hypothesis $\operatorname{inv}\left(C^{k}\right)-\operatorname{inv}\left(C^{k+1}\right)=1$. Let $\sigma_{k}=\phi\left(C^{k}\right)$ and $\sigma_{k+1}=\phi\left(C^{k+1}\right)$. Since $e_{i_{k}}$ and $e_{i_{k}+1}$ are not in the same column of $C^{k}$, we have $\sigma_{k+1}=\sigma_{k} \circ\left(e_{i_{k}}, e_{i_{k}+1}\right)$ in view of Proposition 2.8.

(a) If $e_{i_{k}}$ and $e_{i_{k}+1}$ have the same parity (which is always true except for $i_{k}=n$ ), then the two integers $e_{i_{k}}$ and $e_{i_{k}+1}$ appear in the same subset $\{1,3, \ldots, 2 n+1\}$ or $\{2,4, \ldots, 2 n+2\}$. Consequently, we obtain the two equalities

$$
\begin{aligned}
\sum_{i=1}^{n+1} \sigma_{k+1}(2 i) & =\sum_{i=1}^{n+1} \sigma_{k}(2 i), \\
\left(\operatorname{inv}\left(\sigma_{k+1}^{e}\right)-\operatorname{inv}\left(\sigma_{k}^{e}\right), \operatorname{inv}\left(\sigma_{k+1}^{o}\right)-\operatorname{inv}\left(\sigma_{k}^{o}\right)\right) & =(-1,0) \text { or }(0,-1),
\end{aligned}
$$

thence $\operatorname{st}\left(\sigma_{k+1}\right)=s t\left(\sigma_{k}\right)+1$, which brings Equality $(7)$.

(b) Else $i_{k}=n$ and $\left(e_{i_{k}}, e_{i_{k}+1}\right)=(2 n+2,1)$. From $\sigma_{k+1}=\sigma_{k} \circ\left(e_{i_{k}}, e_{i_{k}+1}\right)$, we obtain

$$
\begin{aligned}
& \sigma_{k+1}^{e}=\sigma_{k}(2) \sigma_{k}(4) \ldots \sigma_{k}(2 n) \sigma_{k}(1), \\
& \sigma_{k+1}^{o}=\sigma_{k}(2 n+2) \sigma_{k}(3) \sigma_{k}(5) \ldots \sigma_{k}(2 n+1) .
\end{aligned}
$$

This provides the three following equations.

$$
\sum_{i=1}^{n+1} \sigma_{k+1}(2 i)=\left(\sum_{i=1}^{n+1} \sigma_{k}(2 i)\right)-\sigma_{k}(2 n+2)+\sigma_{k}(1),
$$




$$
\begin{aligned}
\operatorname{inv}\left(\sigma_{k+1}^{e}\right)=\operatorname{inv}\left(\sigma_{k}^{e}\right)-\mid\left\{2 i<2 n+2 \mid \sigma_{k}(2 i)>\right. & \left.\sigma_{k}(2 n+2)\right\} \mid \\
& +\left|\left\{2 i<2 n+2 \mid \sigma_{k}(2 i)>\sigma_{k}(1)\right\}\right|,
\end{aligned}
$$

$$
\begin{aligned}
\operatorname{inv}\left(\sigma_{k+1}^{o}\right)=\operatorname{inv}\left(\sigma_{k}^{o}\right)-\left|\left\{1<2 i+1 \mid \sigma_{k}(2 i+1)<\sigma_{k}(1)\right\}\right| \\
+\left|\left\{1<2 i+1 \mid \sigma_{k}(2 i+1)<\sigma_{k}(2 n+2)\right\}\right| .
\end{aligned}
$$

We need the following lemma to make explicit Equalities (9) and (10).

Lemma 2.10. We have the equalities

$$
\begin{aligned}
\left|\left\{2 i<2 n+2 \mid \sigma_{k}(2 i)>\sigma_{k}(2 n+2)\right\}\right| & =r_{C^{k}}(2 n+2)+\left(1+(-1)^{\sigma_{k}(2 n+2)}\right) / 2, \\
\left|\left\{2 i<2 n+2 \mid \sigma_{k}(2 i)>\sigma_{k}(1)\right\}\right| & =r_{C^{k}}(1)-\left(1-(-1)^{\sigma_{k}(1)}\right) / 2, \\
\left|\left\{1<2 i+1 \mid \sigma_{k}(2 i+1)<\sigma_{k}(1)\right\}\right| & =l_{C^{k}}(1)+\left(1-(-1)^{\sigma_{k}(1)}\right) / 2, \\
\left|\left\{1<2 i+1 \mid \sigma_{k}(2 i+1)<\sigma_{k}(2 n+2)\right\}\right| & =l_{C^{k}}(2 n+2)-\left(1+(-1)^{\sigma_{k}(2 n+2)}\right) / 2 .
\end{aligned}
$$

Proof. We only demonstrate Equalities (11) and (12), because the proof of (13) is analogous to that of (11) and the proof of (14) is analogous to that of (12).

- Proof of (11): if the dot $e_{i_{k}}=2 n+2$ appears in the $j_{k}$-th column of $C^{k}$, and if the $\operatorname{dot} e_{i-1}=2 i$ (with $1 \leqslant i-1 \leqslant n=i_{k}$ ) appears in the $j_{i-1}$-th column of $C^{k}$, then $\sigma_{k}(2 n+2) \in\left\{2 j_{k}, 2 j_{k}+1\right\}$ and $\sigma_{k}(2 i) \in\left\{2 j_{i-1}, 2 j_{i-1}+1\right\}$. Consequently, the two following assertions are equivalent:

$-\sigma_{k}(2 i)>\sigma_{k}(2 n+2)$;

- either $j_{i-1}>j_{k}$, or $j_{i-1}=j_{k}$ and $\sigma_{k}(2 n+2)=2 j_{i-1}$ (which forces $\sigma_{k}(2 i)$ to be $\left.2 j_{i-1}+1\right)$.

As a result,

$$
\left|\left\{2 i<2 n+2 \mid \sigma_{k}(2 i)>\sigma_{k}(2 n+2)\right\}\right|=r_{C^{k}}(2 n+2)+\delta_{\sigma_{k}(2 n+2)}
$$

where $\delta_{\sigma_{k}(2 n+2)}=1$ if $\sigma_{k}(2 n+2)$ is even, and $\delta_{\sigma_{k}(2 n+2)}=0$ if $\sigma_{k}(2 n+2)$ is odd, i.e., where $\delta_{\sigma_{k}(2 n+2)}=\left(1+(-1)^{\sigma_{k}(2 n+2)}\right) / 2$.

- Proof of (12): with the same reasoning as for (11), we find the equality

$$
\left|\left\{2 i<2 n+2 \mid \sigma_{k}(2 i)>\sigma_{k}(1)\right\}\right|=r_{C^{k}}(1)-1+\left(1+(-1)^{\sigma_{k}(1)}\right) / 2
$$

(with $r_{C^{k}}(1)-1$ instead of $r_{C^{k}}(1)$ because there is an inversion between $1=e_{i_{k+1}}$ and $2 n+2=e_{i_{k}}$, whereas $2 n+2$ is not counted in $\left.\left|\left\{2 i<2 n+2 \mid \sigma_{k}(2 i)>\sigma_{k}(1)\right\}\right|\right)$. Since $-1+\left(1+(-1)^{\sigma_{k}(1)}\right) / 2=-\left(1-(-1)^{\sigma_{k}(1)}\right) / 2$, we obtain $(12)$. 
In view of Lemma 2.10, Equalities (9) and (10) become

$$
\begin{aligned}
& \operatorname{inv}\left(\sigma_{k+1}^{e}\right)-\operatorname{inv}\left(\sigma_{k}^{e}\right)=r_{C^{k}}(1)-r_{C^{k}}(2 n+2)-1+\left((-1)^{\sigma_{k}(1)}-(-1)^{\sigma_{k}(2 n+2)}\right) / 2, \\
& \operatorname{inv}\left(\sigma_{k+1}^{o}\right)-\operatorname{inv}\left(\sigma_{k}^{o}\right)=l_{C^{k}}(2 n+2)-l_{C^{k}}(1)-1+\left((-1)^{\sigma_{k}(1)}-(-1)^{\sigma_{k}(2 n+2)}\right) / 2 .
\end{aligned}
$$

Now, from Lemma 2.6, we know that

$$
\begin{gathered}
\sigma_{k}(1)=y_{n+1+l_{C^{k}}(1)-r_{C^{k}}(1)}, \\
\sigma_{k}(2 n+2)=y_{n+l_{C^{k}}(2 n+2)-r_{C^{k}}(2 n+2) .}
\end{gathered}
$$

From $y_{i}=i+1-(-1)^{i}$ for all $i$, we deduce the two following formulas.

$$
\begin{aligned}
\sigma_{k}(1)=n+2+(-1)^{n}+l_{C^{k}}(1)-r_{C^{k}}(1) & +(-1)^{n+1}\left(1-(-1)^{l_{C^{k}}(1)-r_{C^{k}}(1)}\right), \\
\sigma_{k}(2 n+2)=n+1-(-1)^{n}+l_{C^{k}}(2 n+2) & -r_{C^{k}}(2 n+2) \\
& +(-1)^{n}\left(1-(-1)^{l_{C^{k}}(2 n+2)-r_{C^{k}}(2 n+2)}\right) .
\end{aligned}
$$

By substituting Equalities (17) and (18) in Equalities (8), (15) and (16), we obtain the three new equalities

$$
\begin{aligned}
& \sum_{i=1}^{n+1} \sigma_{k+1}(2 i)-\sum_{i=1}^{n+1} \sigma_{k}(2 i)=1+l_{C^{k}}(1)-l_{C^{k}}(2 n+2)+r_{C^{k}}(2 n+2)-r_{C^{k}}(1) \\
& +(-1)^{n+l_{C^{k}}(1)-r_{C^{k}}(1)}+(-1)^{n+l_{C^{k}}(2 n+2)-r_{C^{k}}(2 n+2)}, \\
& \operatorname{inv}\left(\sigma_{k+1}^{e}\right)-\operatorname{inv}\left(\sigma_{k}^{e}\right)=r_{C^{k}}(1)-r_{C^{k}}(2 n+2)-1 \\
& -\left((-1)^{n+l_{C^{k}}(1)-r_{C^{k}}(1)}+(-1)^{n+l_{C^{k}}(2 n+2)-r_{C^{k}}(2 n+2}\right) / 2, \\
& \operatorname{inv}\left(\sigma_{k+1}^{o}\right)-\operatorname{inv}\left(\sigma_{k}^{o}\right)=l_{C^{k}}(2 n+2)-l_{C^{k}}(1)-1 \\
& -\left((-1)^{n+l_{C^{k}}(1)-r_{C^{k}}(1)}+(-1)^{n+l_{C^{k}}(2 n+2)-r_{C^{k}}(2 n+2)} / 2 .\right.
\end{aligned}
$$

Finally, we obtain Equality (7) by summing Equalities (19), (20) and (21). This proves Theorem 2.2.

Remark 2.4. In [9], the authors proved that $\bar{c}_{n}(q)$ is divisible by $1+q$ if $n$ is odd, but requested a combinatorial proof of this statement. Now, if $n$ is odd, one can prove that every Dellac configuration $C \in D C(n-1)$ is switchable at some even integer, which yields a natural involution $\mathcal{I}$ on $D C(n-1)$ such that $\operatorname{inv}(\mathcal{I}(C))=\operatorname{inv}(C) \pm 1$ for all $C$. This proves combinatorially the divisibility of $\bar{c}_{n}(q)$ by $1+q$ in view of Theorem 2.2 . 


\section{Dellac histories}

\subsection{Weighted Dyck paths}

Recall (see [8]) that a Dyck path $\gamma$ of length $2 n$ is a sequence of points $\left(p_{0}, p_{1}, \ldots, p_{2 n}\right)$ in $\mathbb{N}^{2}$ such that $\left(p_{0}, p_{2 n}\right)=((0,0),(2 n, 0))$, and for all $i \in[2 n]$, the step $\left(p_{i-1}, p_{i}\right)$ is either an up step $(1,1)$ or a down step $(1,-1)$. We denote by $\Gamma(n)$ the set of Dyck paths of length $2 n$. Furthermore, let $\mu=\left(\mu_{n}\right)_{n \geqslant 1}$ be a sequence of elements of a ring. A weighted Dyck path is a Dyck path $\gamma=\left(p_{i}\right)_{0 \leqslant i \leqslant 2 n} \in \Gamma(n)$ whose each up step has been weighted by 1 , and each down step $\left(p_{i-1}, p_{i}\right)$ from height $h$ (i.e., such that $\left.p_{i-1}=(i-1, h)\right)$ has been weighted by $\mu_{h}$.

The weight

$$
\omega_{\mu}(\gamma)
$$

of the weighted Dyck path $\gamma$ is the product of the weights of all steps.

Remark 3.1. If $\gamma=\left(p_{i}\right)_{0 \leqslant i \leqslant 2 n} \in \Gamma(n)$, then $p_{i}=\left(i, n_{u}(i)-n_{d}(i)\right)$ where $n_{u}(i)$ and $n_{d}(i)$ are defined as the numbers of up steps and down steps on the left of $p_{i}$ respectively (in particular $\left.n_{u}(i)+n_{d}(i)=i\right)$. Consequently, since the final point of $\gamma$ is $p_{2 n}=(2 n, 0)$, the path $\gamma$ has exactly $n$ up steps and $n$ down steps, and for all $j \in[n]$, the points $p_{2 j-1}$ and $p_{2 j}$ are at heights respectively odd and even.

Definition 3.1 (Labelled steps). Let $\gamma=\left(p_{i}\right)_{0 \leqslant i \leqslant 2 n} \in \Gamma(n)$. For all $i \in[n]$, we denote by $s_{i}^{u}(\gamma)$ (resp. $s_{i}^{d}(\gamma)$ ) the $i$-th up step (resp. down step) of $\gamma$. When there is no ambiguity, we write $s_{i}^{u}$ and $s_{i}^{d}$ instead of $s_{i}^{u}(\gamma)$ and $s_{i}^{d}(\gamma)$.

Remark 3.2. If $s_{i}^{u}(\gamma)=\left(p_{2 j-2}, p_{2 j-1}\right)$ or $\left(p_{2 j-1}, p_{2 j}\right)$ where $p_{2 j-2}=(2 j-2,2 k)$ for some $k \geqslant 0$, then, following Remark 3.1, we know that $2 k=n_{u}(2 j-2)-n_{d}(2 j-2)=$ $2 n_{u}(2 j-2)-(2 j-2)$, and by definition of $s_{i}^{u}(\gamma)$ it is necessary that $n_{u}(2 j-2)=i-1$, and we obtain $2 k=2(i-j)$ hence $i=j+k$. In the same context, if $s_{i}^{d}(\gamma)=\left(p_{2 j-1}, p_{2 j}\right)$ or $\left(p_{2 j-2}, p_{2 j-1}\right)$, then we obtain $i=j-k$ by an analogous reasoning.

\subsection{Dellac histories}

Definition 3.2. A Dellac history of length $2 n$ is a pair $(\gamma, \xi)$ where $\gamma=\left(p_{i}\right)_{0 \leqslant i \leqslant 2 n} \in \Gamma(n)$ and $\xi=\left(\xi_{1}, \xi_{2}, \ldots, \xi_{n}\right)$ where $\xi_{i}$ is a pair of nonnegative integers $\left(n_{1}(i), n_{2}(i)\right)$ with the following conditions. Let $j \in[n]$ be such that the $i$-th down step $s_{i}^{d}$ of $\gamma$ is one the two steps $\left(p_{2 j-2}, p_{2 j-1}\right)$ and $\left(p_{2 j-1}, p_{2 j}\right)$, and let $2 k$ be the height of $p_{2 j-2}$. There are three cases.

1. If $s_{i}^{d}=\left(p_{2 j-2}, p_{2 j-1}\right)$ such that $\left(p_{2 j-1}, p_{2 j}\right)$ is an up step (see Figure $\left.8,(1)\right)$, then

$$
k \geqslant n_{1}(i)>n_{2}(i) \geqslant 0,
$$

and we attach a weight $\omega_{i}=q^{2 k-n_{1}(i)-n_{2}(i)}$ to $s_{i}^{d}$. 
2. If $s_{i}^{d}=\left(p_{2 j-1}, p_{2 j}\right)$ such that $\left(p_{2 j-2}, p_{2 j-1}\right)$ is an up step (see Figure 8,(2)), then

$$
0 \leqslant n_{1}(i) \leqslant n_{2}(i) \leqslant k,
$$

and we attach a weight $\omega_{i}=q^{2 k-n_{1}(i)-n_{2}(i)}$ to $s_{i}^{d}$.

3. If $\left(p_{2 j-2}, p_{2 j-1}\right)$ and $\left(p_{2 j-1}, p_{2 j}\right)$ are both down steps (see Figure 8,(3)), we can suppose that $s_{i}^{d}=\left(p_{2 j-2}, p_{2 j-1}\right)$ and $s_{i+1}^{d}=\left(p_{2 j-1}, p_{2 j}\right)$, then

$$
k-1 \geqslant n_{1}(i) \geqslant n_{2}(i) \geqslant 0,
$$

and we attach a weight $\omega_{i}=q^{2 k-1-n_{1}(i)-n_{2}(i)}$ to $s_{i}^{d}$, also

$$
0 \leqslant n_{1}(i+1) \leqslant n_{2}(i+1) \leqslant k-1,
$$

and we attach a weight $\omega_{i+1}=q^{2 k-2-n_{1}(i+1)-n_{2}(i+1)}$ to $s_{i+1}^{d}$.

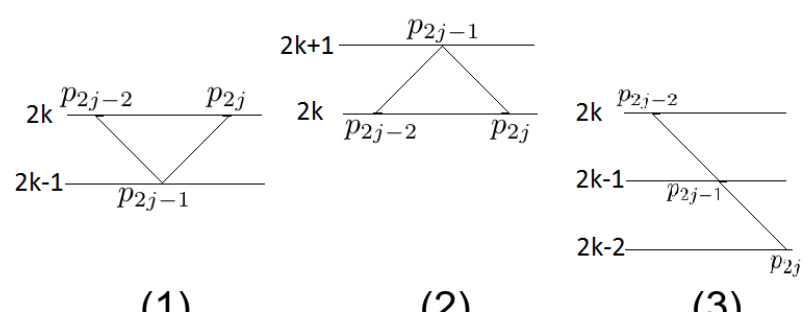

(1)

(2)

(3)

Figure 8:

The weight $\omega(\gamma, \xi)$ of the history $(\gamma, \xi)$ is the product of the weights of all down steps. We denote by $D H(n)$ the set of Dellac histories of length $2 n$.

Prior to connecting Dellac histories to weighted Dyck paths, one can easily verify the two following results.

Lemma 3.1. For all $p \geqslant 1$, the polynomial

$$
\sum_{0 \leqslant n_{1} \leqslant n_{2} \leqslant p-1} q^{2 p-2-n_{1}-n_{2}}
$$

is the q-binomial coefficient $\left\{\begin{array}{c}p+1 \\ 2\end{array}\right\}_{q}=\left(1-q^{p+1}\right)\left(1-q^{p}\right) /\left(\left(1-q^{2}\right)(1-q)\right)$.

Proposition 3.2. For all $\gamma_{0} \in \Gamma(n)$, we have the equality

$$
\sum_{\left(\gamma_{0}, \xi\right) \in D H(n)} \omega\left(\gamma_{0}, \xi\right)=\omega_{\lambda}\left(\gamma_{0}\right)
$$

where $\omega_{\lambda}$ has been defined in (22), and where $\lambda=\left(\lambda_{n}\right)_{n \geqslant 1}$ is the sequence defined in Theorem 1.2. 
Proof. By Definition 3.2, the polynomial $\sum_{\left(\gamma_{0}, \xi\right) \in D H(n)} \omega\left(\gamma_{0}, \xi\right)$ is the product of the weights of all down steps of $\gamma_{0}$, where the weight of a down step $s_{i}^{d}\left(\gamma_{0}\right)$ is a sum of monomials over pairs of nonnegative integers with conditions depending on the three cases 1.,2. or 3 . in which $s_{i}^{d}\left(\gamma_{0}\right)$ may be found. If $s_{i}^{d}\left(\gamma_{0}\right)$ is a down step $\left(p_{2 j-1}, p_{2 j}\right)$ from height $2 k-1$ in the case 3 ., then it is weighted by the polynomial $\sum_{0 \leqslant n_{1} \leqslant n_{2} \leqslant k-1} q^{2 k-2-n_{1}-n_{2}}$, which, in view of Lemma 3.1, equals $\left\{\begin{array}{c}k+1 \\ 2\end{array}\right\}_{q}=\lambda_{2 k-1}$. If $s_{i}^{d}\left(\gamma_{0}\right)$ is a down step $\left(p_{2 j-2}, p_{2 j-1}\right)$ from height $2 k$, still in the case 3 ., then it is weighted by the polynomial $\sum_{k-1 \geqslant n_{1} \geqslant n_{2} \geqslant 0} q^{2 k-1-n_{1}-n_{2}}=$ $q\left\{\begin{array}{c}k+1 \\ 2\end{array}\right\}_{q}=\lambda_{2 k}$. If $s_{i}^{d}\left(\gamma_{0}\right)$ is a down step $\left(p_{2 j-1}, p_{2 j}\right)$ from height $2 k+1$ in the case 2 ., then it is weighted by the polynomial $\sum_{0 \leqslant n_{1} \leqslant n_{2} \leqslant k} q^{2 k-n_{1}-n_{2}}=\left\{\begin{array}{c}k+2 \\ 2\end{array}\right\}_{q}=\lambda_{2 k+1}$. Finally, if $s_{i}^{d}\left(\gamma_{0}\right)$ is a down step $\left(p_{2 j-2}, p_{2 j-1}\right)$ from height $2 k$ in the case 1 ., then it is weighted by the polynomial $\sum_{k \geqslant n_{1}>n_{2} \geqslant 0} q^{2 k-n_{1}-n_{2}}$, which, by setting $m_{1}=n_{1}-1$ and $m_{2}=n_{2}$, equals $\sum_{k-1 \geqslant m_{1} \geqslant m_{2} \geqslant 0} q^{2 k-1-m_{1}-m_{2}}=q\left\{\begin{array}{c}k+1 \\ 2\end{array}\right\}=\lambda_{2 k}$ in view of Lemma 3.1.

Following Proposition 3.2, we have

$$
\sum_{(\gamma, \xi) \in D H(n)} \omega(\gamma, \xi)=\sum_{\gamma \in \Gamma(n)} \omega_{\lambda}(\gamma)
$$

for all $n \geqslant 0$. Therefore, from a well-known result due to Flajolet [8], the generating function $\sum_{n \geqslant 0}\left(\sum_{(\gamma, \xi) \in D H(n)} \omega(\gamma, \xi)\right) t^{n}$ is the continued fraction expansion of Formula (4). Consequently, to demonstrate Theorem 1.4, it suffices to prove that $\tilde{h}_{n}(q)=$ $\sum_{(\gamma, \xi) \in D H(n)} \omega(\gamma, \xi)$, which is a straight corollary of the following theorem.

Theorem 3.3. There exists a bijective map $\Phi: D C(n) \rightarrow D H(n)$ such that

$$
\omega(\Phi(C))=q^{\left(\begin{array}{l}
n \\
2
\end{array}\right)-i n v(C)}
$$

for all $C \in D C(n)$.

\subsection{Proof of Theorem 3.3}

In this part, we give preliminaries and connections between Dellac configurations and Dyck paths. Then, we define the map $\Phi: D C(n) \rightarrow D H(n)$ and we demonstrate the statistic preservation formula (23). Finally, we prove that $\Phi$ is bijective by giving a map $\Psi: D H(n) \rightarrow D C(n)$ which happens to be $\Phi^{-1}$.

\subsubsection{Preliminaries on Dellac configurations}

Definition 3.3. Let $C \in D C(n)$. If $i \leqslant n$, we denote by $l_{C}^{e}\left(e_{i}\right)$ the number of inversions of $C$ between $e_{i}$ and any even $\operatorname{dot} e_{i^{\prime} \leqslant n}$ with $i^{\prime}>i$. In the same way, if $i>n$, we denote by $r_{C}^{o}\left(e_{i}\right)$ the number of inversions of $C$ between $e_{i}$ and any odd $\operatorname{dot} e_{i^{\prime}>n}$ with $i^{\prime}<i$.

Definition 3.4. Let $C \in D C(n)$ and $j \in[n]$. We define the height $h(j)$ of the integer $j$ as the number $n_{e}(j)-n_{o}(j)$ where $n_{e}(j)$ (resp. $n_{o}(j)$ ) is the number of even dots (resp. odd dots) in the first $j-1$ columns of $C$ (with $\left.n_{e}(1)=n_{o}(1)=0\right)$. 
Remark 3.3. Since the first $j-1$ columns of $C$ contain exactly $2 j-2$ dots and, from Remark 2.1, always contain the $j-1$ even dots $e_{1}, e_{2}, \ldots, e_{j-1}$, there exists $k \in\{0,1, \ldots, j-1\}$ such that $n_{e}(j)=j-1+k$ and $n_{o}(j)=j-1-k$. In particular $h(j)=2 k$.

Lemma 3.4. Let $C \in D C(n)$, let $j \in[n]$ and $k \geqslant 0$ such that $h(j)=2 k$. If the $j$-th column of $C$ contains two odd dots, there exists $j^{\prime}<j$ such $h\left(j^{\prime}+1\right)=2 k$ and such that the $j^{\prime}$-th column of $C$ contains two even dots.

Proof. From Remark 3.3, we have $n_{e}(j)=j-1+k$ and $n_{o}(j)=j-1-k$. Since the only $j$ odd dots that the first $j$ columns may contain are $e_{n+1}, e_{n+2}, \ldots, e_{n+j-1}, e_{n+j}$, and since the $j$-th column already contains two odd dots, the first $j-1$ columns contain at most $j-2$ odd dots. In other words, since they contain $n_{o}(j)=j-1-k$ odd dots, we obtain $k \geqslant 1$. Thus $h(j)=2 k>0$. Since $h(1)=0$, there exists $j^{\prime} \in[j-1]$ such that $h\left(j^{\prime}+1\right)=2 k$ and $h\left(j^{\prime}\right)<2 k$. Obviously $h\left(j^{\prime}+1\right)-h\left(j^{\prime}\right) \in\{-2,0,2\}$, so $h\left(j^{\prime}\right)=2 k-2$ and the $j^{\prime}$-th column of $C$ contains two even dots.

\subsection{2 $\quad$ Map $\Phi: D C(n) \rightarrow D H(n)$}

Definition $3.5(\boldsymbol{\Phi})$. Let $C \in D C(n)$, we define $\Phi(C)$ as $(\gamma, \xi)$, where $\gamma=\left(p_{i}\right)_{0 \leqslant i \leqslant 2 n}$ (which is a path in $\mathbb{Z}^{2}$ whose initial point $p_{0}$ is defined as $\left.(0,0)\right)$ and $\xi=\left(\xi_{1}, \ldots, \xi_{n}\right.$ ) (which is a sequence of pairs of nonnegative integers) are provided by the following algorithm. For $j=1$ to $n$, let $e_{i_{1}(j)}$ and $e_{i_{2}(j)}$ (with $\left.i_{1}(j)<i_{2}(j)\right)$ be the two dots of the $j$-th column of $C$.

1. If $i_{2}(j) \leqslant n$, then $\left(p_{2 j-2}, p_{2 j-1}\right)$ and $\left(p_{2 j-1}, p_{2 j}\right)$ are defined as up steps.

2. If $i_{1}(j) \leqslant n<i_{2}(j)$, let $i \in[n]$ such that $i-1$ down steps have already been defined. We define $\xi_{i}$ as $\left(l_{C}^{e}\left(e_{i_{1}(j)}\right), r_{C}^{o}\left(e_{i_{2}(j)}\right)\right.$. Afterwards,

(a) if $l_{C}^{e}\left(e_{i_{1}(j)}\right)>r_{C}^{o}\left(e_{i_{2}(j)}\right)$, we define $\left(p_{2 j-2}, p_{2 j-1}\right)$ as a down step and $\left(p_{2 j-1}, p_{2 j}\right)$ as an up step (see Figure 8,(1));

(b) if $l_{C}^{e}\left(e_{i_{1}(j)}\right) \leqslant r_{C}^{o}\left(e_{i_{2}(j)}\right)$, we define $\left(p_{2 j-2}, p_{2 j-1}\right)$ as an up step and $\left(p_{2 j-1}, p_{2 j}\right)$ as a down step (see Figure 8,(2)).

3. If $n<i_{1}(j)$, let $i \in[n]$ such that $i-1$ down steps have already been defined. We define $\left(p_{2 j-2}, p_{2 j-1}\right)$ and $\left(p_{2 j-1}, p_{2 j}\right)$ as down steps (see Figure 8,(3)). Afterwards, let $k \geqslant 0$ such that $p_{2 j-2}=(2 j-2,2 k)$. Obviously, the number $n_{u}(2 j-2)=j-1+k$ of up steps (resp. the number $n_{d}(2 j-2)=j-1-k$ of down steps) that have already been defined is the number $n_{e}(j)$ of even dots (resp. the number $n_{o}(j)$ of odd dots) in the first $j-1$ columns of $C$, thence $h(j)=2 k$. From Lemma 3.4, there exists $j^{\prime}<j$ such that $h\left(j^{\prime}+1\right)=2 k$ (which means $\left.p_{2 j^{\prime}}=\left(2 j^{\prime}, 2 k\right)\right)$ and such that the $j^{\prime}$-th column of $C$ contains two even dots, which means $\left(p_{2 j^{\prime}-2}, p_{2 j^{\prime}-1}\right)$ and $\left(p_{2 j^{\prime}-1}, p_{2 j^{\prime}}\right)$ are two consecutive up steps (see Figure 9). Now, we consider the maximum $j_{m}<j$ of the integers $j^{\prime}$ that verify this property, and we consider the two dots $e_{i_{1}\left(j_{m}\right)}$ and 


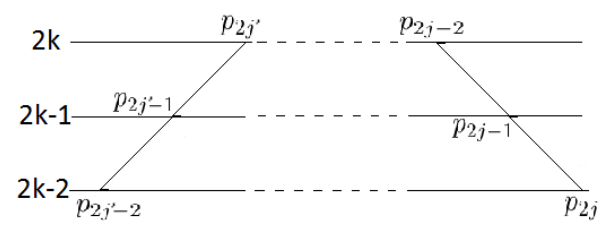

Figure 9: Two consecutive up steps and down steps at the same level.

$e_{i_{2}\left(j_{m}\right)}$ (with $i_{1}\left(j_{m}\right)<i_{2}\left(j_{m}\right)$ ) of the $j_{m}$-th column of $C$. Finally, we define $\xi_{i}$ and $\xi_{i+1}$ as

$$
\begin{aligned}
\xi_{i} & =\left(l_{C}^{e}\left(e_{i_{1}\left(j_{m}\right)}\right), l_{C}^{e}\left(e_{i_{2}\left(j_{m}\right)}\right)\right), \\
\xi_{i+1} & =\left(r_{C}^{o}\left(e_{i_{1}(j)}\right), r_{C}^{o}\left(e_{i_{2}(j)}\right)\right) .
\end{aligned}
$$

Example 3.1. The Dellac configuration $C \in D C(6)$ of Figure 10 yields the data $\Phi(C)=$ $(\gamma, \xi)$, which is in fact a Dellac history, depicted in Figure 11 (since $\Phi(C)$ is a Dellac history, we have indicated the weight $\omega_{i}$ of the $i$-th down step $s_{i}^{d}$ of $\gamma$ for all $i \in[6]$, see Definition 3.2).

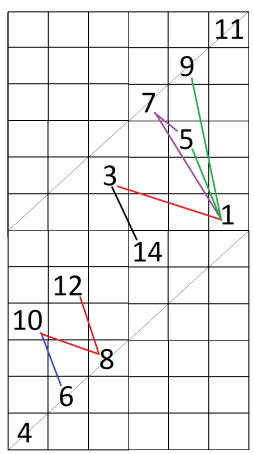

Figure 10: $C \in D C(6)$.

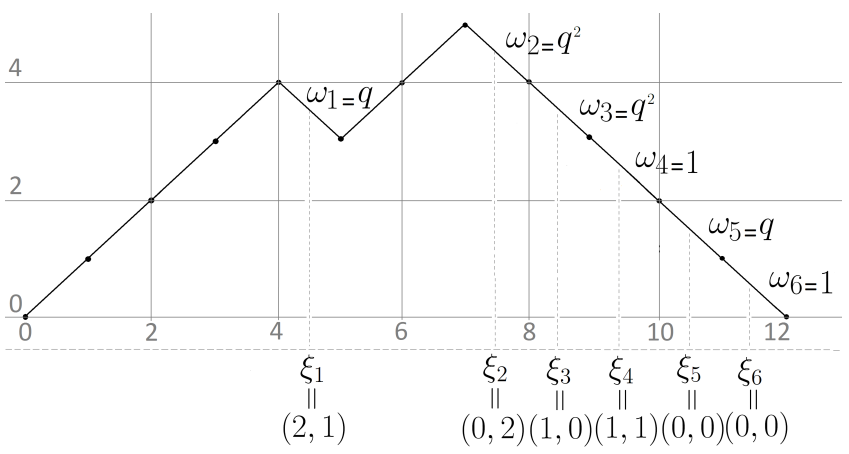

Figure 11: $\Psi(C) \in D H(6)$.

Remark 3.4. If $\Phi(C)=(\gamma, \xi)$, let $j \in[n]$, the number of up steps (resp. down steps) among the first $2 j$ steps of $\gamma$, is in fact the number of even dots (resp. odd dots) in the first $j$ columns of $C$. With precision, for all $i \in[n]$, the even $\operatorname{dot} e_{p_{C}(i)}$ and the odd dot $e_{n+q_{C}(i)}$ (see Definition 2.3) give birth to the $i$-th up step and the $i$-th down step of $\gamma$ respectively. In particular, the path $\gamma$ has $n$ up steps and $n$ down steps, so $p_{2 n}=(2 n, 0)$. To prove that $\gamma$ is a Dyck path, we still have to check that it never goes below the line $y=0$.

Remark 3.5. In the context (3) of Definition 3.5, if $h(j)=2 k$ (i.e., if $p_{2 j-2}=(2 j-2,2 k)$ ), then the maximum $j_{m}$ of the integers $j^{\prime}<j$ such that $h\left(j^{\prime}+1\right)=2 k$ and such that the $j^{\prime}$-th column contains two even dots, is such that $\left(p_{2 j_{m}-2}, p_{2 j_{m}-1}\right)$ and $\left(p_{2 j_{m}-1,2 j_{m}}\right)$ are the last two consecutive up steps from level $2 k-2$ towards level $2 k$ in $\gamma$.

Proposition 3.5. Let $C \in D C(n)$ and $(\gamma, \xi)=\Phi(C)$. The path $\gamma$ is a Dyck path. 
Proof. From Remark 3.4, it suffices to prove that $\gamma=\left(p_{0}, p_{1}, \ldots, p_{2 n}\right)$ never goes below the line $y=0$. If we suppose the contrary, there exists $i_{0} \in\{0,1, \ldots, 2 n-1\}$ such that $p_{i_{0}}=\left(i_{0}, 0\right)$ and $\left(p_{i_{0}}, p_{i_{0}+1}\right)$ is a down step. From Remark 3.1, we know that $p_{i_{0}}=\left(i_{0}, 0\right)=\left(i_{0}, 2 n_{u}\left(i_{0}\right)-i_{0}\right)$, so $i_{0}=2 n_{u}\left(i_{0}\right)$. Let $j_{0}=n_{u}\left(i_{0}\right)+1 \in[n]$. In the first $j_{0}-1$ columns of $C$, from Remark 3.4, there are $n_{u}\left(i_{0}\right)=j_{0}-1$ even dots and $n_{d}\left(i_{0}\right)=j_{0}-1$ odd dots. Consequently, since those first $j_{0}-1$ columns always contain the $j_{0}-1$ even dots $e_{1}, e_{2}, \ldots, e_{j_{0}-1}$ and cannot contain any other odd dot than $e_{n+1}, e_{n+2}, \ldots, e_{n+j_{0}-1}$ (see Remark 2.1), the $2 j_{0}-2$ dots they contain are precisely $e_{1}, e_{2}, \ldots, e_{j_{0}-1}$ and $e_{n+1}, e_{n+2}, \ldots, e_{n+j_{0}-1}$. Therefore, the only two dots that the $j_{0}$-th column may contain are $e_{j_{0}}$ and $e_{n+j_{0}}$. But then, it forces $l_{C}^{e}\left(e_{j_{0}}\right)$ and $r_{C}^{o}\left(e_{n+j_{0}}\right)$ to equal 0 . In particular $l_{C}^{e}\left(e_{j_{0}}\right) \leqslant r_{C}^{o}\left(e_{n+j_{0}}\right)$. Following the case $2(b)$ of Definition 3.5 , it means $\left(p_{i_{0}}, p_{i_{0}+1}\right)$ is defined as an up step, which is absurd by hypothesis.

Proposition 3.6. For all $C \in D C(n)$, the data $\Phi(C)$ is a Dellac history of length $2 n$.

Proof. Let $\Phi(C)=(\gamma, \xi)=\left(\left(p_{0}, p_{1}, \ldots, p_{2 n}\right),\left(\xi_{1}, \xi_{2}, \ldots, \xi_{n}\right)\right)$. We know that $\gamma \in \Gamma(n)$. It remains to prove that $\xi$ fits the appropriate inequalities described in Definition 3.2. Let $j \in[n]$ and let $e_{i_{1}(j)}$ and $e_{i_{2}(j)}$ (with $j \leqslant i_{1}(j)<i_{2}(j) \leqslant j+n$ ) be the two dots of the $j$-th column of $C$.

- If $\left(p_{2 j-1}, p_{2 j}\right)$ is the down step $s_{i}^{d}$ in the context $2(a)$ of Definition 3.5 , then $\xi_{i}=$ $\left.\left(n_{1}, n_{2}\right)=\left(l_{C}^{e}\left(e_{i_{1}(j)}\right)\right), r_{C}^{o}\left(e_{i_{2}(j)}\right)\right)$ with $l_{C}^{e}\left(e_{i_{1}(j)}\right)>r_{C}^{o}\left(e_{i_{2}(j)}\right)$. Here, the appropriate inequality to check is $k \geqslant n_{1}>n_{2}$ (this is the context 1. of Definition 3.2). Since the first $j-1$ columns of $C$ contain $j-1+k$ even dots, including the $j-1$ dots $e_{1}, e_{2}, \ldots, e_{j-1}$ (with $j-1<i_{1}(j)$ ), there is no inversion between any of these dots and $e_{i_{1}(j)}$. Consequently, in the first $j-1$ columns of $C$, there are at most $(j-1+k)-(j-1)=k$ even $\operatorname{dots} e_{i}$ with $n \geqslant i>i_{1}(j)$, thence $n_{1}=l_{C}^{e}\left(e_{i_{1}(j)}\right) \leqslant k$.

- Similarly, if $\left(p_{2 j-2}, p_{2 j-1}\right)$ is the down step $s_{i}^{d}$ set in the context $2(b)$ of Definition 3.5 , then we have $\left.\xi_{i}=\left(n_{1}, n_{2}\right)=\left(l_{C}^{e}\left(e_{i_{1}(j)}\right)\right), r_{C}^{o}\left(e_{i_{2}(j)}\right)\right)$, with $l_{C}^{e}\left(e_{i_{1}(j)}\right) \leqslant r_{C}^{o}\left(e_{i_{2}(j)}\right)$. Now, the appropriate equality to check is $n_{1} \leqslant n_{2} \leqslant k$ (this is the context 2 . of Definition 3.2). The first $j$ columns of $C$ contain $j-k$ odd dots and the $i_{2}(j)-n$ lines from the $(n+1)$-th line to the $i_{2}(j)$-th line contain $i_{2}(j)-n$ odd dots, so, in the $n-j$ last columns, the number of odd dots $e_{i}$ with $n<i<i_{2}(j)$ is at most $\left(i_{2}(j)-n\right)-(j-k)=k+\left(i_{2}(j)-j-n\right) \leqslant k$, thence $n_{2}=r_{C}^{o}\left(e_{i_{2}(j)}\right) \leqslant k$.

- Finally, if $\left(p_{2 j-2}, p_{2 j-1}\right)$ and $\left(p_{2 j-1}, p_{2 j}\right)$ are two consecutive down steps $s_{i}^{d}$ and $s_{i+1}^{d}$ in the context 3. of Definition 3.5, then

$$
\begin{aligned}
\xi_{i} & =\left(l_{C}^{e}\left(e_{i_{1}\left(j_{m}\right)}\right), l_{C}^{e}\left(e_{i_{2}\left(j_{m}\right)}\right)\right), \\
\xi_{i+1} & =\left(r_{C}^{o}\left(e_{i_{1}(j)}\right), r_{C}^{o}\left(e_{i_{2}(j)}\right)\right)
\end{aligned}
$$

and the two inequalities to check (this is the context 3. of Definition 3.2) are:

$$
\begin{aligned}
k-1 & \geqslant l_{C}^{e}\left(e_{i_{1}\left(j_{m}\right)}\right) \geqslant l_{C}^{e}\left(e_{i_{2}\left(j_{m}\right)}\right), \\
r_{C}^{o}\left(e_{i_{1}(j)}\right) & \leqslant r_{C}^{o}\left(e_{i_{2}(j)}\right) \leqslant k-1 .
\end{aligned}
$$


- Proof of $(24)$ : since $i_{1}\left(j_{m}\right)<i_{2}\left(j_{m}\right)$, obviously $l_{C}^{e}\left(e_{i_{1}\left(j_{m}\right)}\right) \geqslant l_{C}^{e}\left(e_{i_{2}\left(j_{m}\right)}\right)$. Afterwards, since $p_{2 j_{m}-2}$ is at the level $h\left(j_{m}\right)=2 k-2$, there are $j_{m}-1+(k-1)=$ $j_{m}+k-2$ even dots in the first $j_{m}-1$ columns of $C$. Since the first $j_{m}-1$ rows of $C$ contain the $j_{m}-1$ even dots $e_{1}, e_{2}, \ldots, e_{j_{m}-1}$, the first $j_{m}-1$ columns of $C$ contain at most $\left(j_{m}+k-2\right)-\left(j_{m}-1\right)=k-1$ even dots $e_{i}$ with $n \geqslant i>i_{1}\left(j_{m}\right)$, thence $l_{C}^{e}\left(e_{i_{1}\left(j_{m}\right)}\right) \leqslant k-1$.

- Proof of $(25)$ : since $i_{1}(j)<i_{2}(j)$, obviously $r_{C}^{o}\left(e_{i_{1}(j)}\right) \leqslant r_{C}^{o}\left(e_{i_{2}(j)}\right)$. Afterwards, since $p_{2 j}$ is at the level $h(j+1)=2 k-2$, there are $j-(k-1)=j-k+1$ odd dots in the first $j$ columns of $C$. Since the $j$ rows, from the $(n+1)$-th row to the $(n+j)$-th row of $C$, contain $j$ odd dots, the $n-j$ last columns of $C$ contain at most $j-(j-k+1)=k-1$ odd dots $e_{i}$ with $n<i<i_{2}\left(j_{m}\right)$, thence $r_{C}^{o}\left(e_{i_{2}(j)}\right) \leqslant k-1$.

So $\Phi(C)$ is a Dellac history of length $n$.

\subsubsection{Proof of the statistic preservation formula (23)}

Let $C \in D C(n)$ and $\Phi(C)=(\gamma, \xi)$ with $\gamma=\left(p_{0}, p_{1}, \ldots, p_{2 n}\right)$ and $\xi=\left(\xi_{1}, \xi_{2}, \ldots, \xi_{2 n}\right)$. By definition, we have $\omega(\Phi(C))=\prod_{i=1}^{n} \omega_{i}$ where $\omega_{i}$ is the weight of the $i$-th down step $s_{i}^{d}$ of $\gamma$. In the contexts 1 . or 2. of Definition 3.2, we have

$$
\omega_{i}=q^{2 k-l_{C}^{e}\left(e_{i_{1}(j)}\right)-r_{C}^{o}\left(e_{i_{2}(j)}\right)} .
$$

Since $p_{2 j-2}$ is at the level $h(j)=2 k$, the first $j-1$ columns of $C$ contain $j-1-k$ odd dots. Consequently, following Definition 3.5, the step $s_{i}^{d}$ is the $(j-k)$-th down step of $\gamma$, i.e., the integer $i$ equals $j-k$. Also, since the first $j$ columns of $C$ contain $j+k$ even dots, the last $n-j$ columns of $C$ (from the $(j+1)$-th column to the $n$-th column) contain $n-(j+k)=n-j-k=i-k$ even dots. As a result, we obtain the equality

$$
r_{C}\left(e_{i_{2}(j)}\right)=r_{C}^{o}\left(e_{i_{2}(j)}\right)+i-k
$$

In view of (27), Equality (26) becomes $\omega_{i}=q^{n-i-\left(l_{C}^{e}\left(e_{i_{1}(j)}\right)+r_{C}\left(e_{i_{2}(j)}\right)\right)}$. With the same reasoning, if $s_{i}^{d}$ and $s_{i+1}^{d}$ are two consecutive down steps in the context 3 . of Definition 3.2 , then by commuting factors of $\omega_{i}$ and $\omega_{i+1}$, we obtain the equality

$$
\omega_{i} \omega_{i+1}=\left(q^{n-i-\left(l_{C}^{e}\left(e_{i_{1}\left(j_{m}\right)}\right)+r_{C}\left(e_{i_{2}\left(j_{m}\right)}\right)\right)}\right)\left(q^{n-(i+1)-\left(l_{C}^{e}\left(e_{i_{1}(j)}\right)+r_{C}\left(e_{i_{2}(j)}\right)\right)}\right) .
$$

From $\omega\left(\Phi(C)=\prod_{i=1}^{n} \omega_{i}\right.$, it follows that

$$
\omega(\Phi(C))=q^{\left(\sum_{i=1}^{n} n-i\right)-\left(\sum_{i \leqslant n} l_{C}^{e}\left(e_{i}\right)+\sum_{i>n} r_{C}\left(e_{i}\right)\right)} .
$$

Now, it is easy to see that $\operatorname{inv}(C)=\sum_{i \leqslant n} l_{C}^{e}\left(e_{i}\right)+\sum_{i>n} r_{C}\left(e_{i}\right)$. In view of the latter remark, Formula (28) becomes Formula (23). 


\subsubsection{Proof of the bijectivity of $\Phi: D C(n) \rightarrow D H(n)$}

To end the proof of Theorem 3.3, it remains to show that $\Phi$ is bijective. To this end, we construct (in Definition 3.6) a map $\Psi: D H(n) \rightarrow D C(n)$ and we prove in Lemma 3.8 that $\Phi$ and $\Psi$ are inverse maps.

Definition 3.6. Let $S=(\gamma, \xi) \in D H(n)$ with $\gamma=\left(p_{0}, p_{1}, \ldots, p_{2 n}\right)$ and $\xi=\left(\xi_{1}, \ldots, \xi_{n}\right)$. We define $\Psi(S)$ as a tableau $T$ of width $n$ and height $2 n$, in which we insert the $2 n$ dots $e_{1}, e_{2}, \ldots, e_{2 n}$ according to the two following (analogous and independant) algorithms.

1. Insertion of the $\boldsymbol{n}$ odd $\operatorname{dots} \boldsymbol{e}_{\boldsymbol{n}+\mathbf{1}}, \boldsymbol{e}_{\boldsymbol{n}+\mathbf{2}}, \ldots, \boldsymbol{e}_{\mathbf{2} \boldsymbol{n}}$. Let $\mathcal{I}_{0}^{o}=(1,2, \ldots, n)$. For $i=1$ to $n$, consider $j_{i} \in[n]$ such that the $i$-th down step $s_{i}^{d}$ of $\gamma$ is one of the two steps $\left(p_{2 j_{i}-2}, p_{2 j_{i}-1}\right)$ or $\left(p_{2 j_{i}-1}, p_{2 j_{i}}\right)$. If the sequence $\mathcal{I}_{i-1}^{o}$ is defined, we denote by $H(i)$ the hypothesis " $\mathcal{I}_{i-1}^{o}$ has length $n+1-i$ such that for all $j \in\{i, i+1, \ldots, n\}$, the $(j-i+1)$-th element of $\mathcal{I}_{i-1}^{o}$ is inferior to $n+j$ ". If the hypothesis $H(i+1)$ is true, then we iterate the algorithm to $i+1$. At the beginning, the sequence $\mathcal{I}_{0}^{o}$ is defined and $H(1)$ is obviously true so we can initiate the algorithm.

(a) If $s_{i}^{d}$ is a down step in the context 1. or 2. of Definition 3.2, let $\left(n_{1}, n_{2}\right)=\xi_{i}$. In particular, since $n_{2} \leqslant k=j_{i}-i$ (see Remark 3.2) and $j_{i} \leqslant n$, we have $1+n_{2} \leqslant n-i+1$ so, from Hypothesis $H(i)$, we can consider the $\left(1+n_{2}\right)$-th element of $\mathcal{I}_{i-1}^{o}$, say, the integer $q$. We insert the odd dot $e_{n+q}$ in the $j_{i}$-th column of $T$. From Hypothesis $H(i)$, the $\left(j_{i}-i+1\right)$-th element of $\mathcal{I}_{i-1}^{o}$ is inferior to $n+j_{i}$, and $1+n_{2} \leqslant 1+k=j_{i}-i+1$. Consequently, the dot $e_{n+q}$ is between the lines $y=x$ and $y=x+n$. Afterwards, we define $\mathcal{I}_{i}^{o}$ as the sequence $\mathcal{I}_{i-1}^{o}$ from which we have removed $q$ (by abusing the notation, we write $\left.\mathcal{I}_{i}^{o}:=\mathcal{I}_{i-1}^{o} \backslash\{q\}\right)$. Thus, the sequence $\mathcal{I}_{i}^{o}$ has length $n+1-(i+1)$. Also, if $j \in\{i+1, i+2, \ldots, n\}$, then following Hypothesis $H(i)$, the $(j-i)$-th element of $\mathcal{I}_{i-1}^{o}$ is inferior to $n+j-1$, so the $(j-(i+1)+1)$-th element of $\mathcal{I}_{i}^{o}$ is inferior to $n+j-1<n+j$. Therefore, Hypothesis $H(i+1)$ is true and we can iterate the algorithm to $i+1$.

(b) If $s_{i}^{d}$ and $s_{i+1}^{d}$ are two consecutive down steps in the context 3 . of Definition 3.2 , let $\left(n_{1}, n_{2}\right)=\xi_{i+1}$. In particular $n_{1} \leqslant n_{2} \leqslant k-1=j_{i}-i-1 \leqslant n-i-1$, so $1+n_{1}<2+n_{2} \leqslant j_{i}-i+1$. Consequently, following Hypothesis $H(i)$, we can consider the $\left(1+n_{1}\right)$-th element of $\mathcal{I}_{i-1}^{o}$, say, the integer $q_{1}$, and the $\left(2+n_{2}\right)$-th element of $\mathcal{I}_{i-1}^{o}$, say, the integer $q_{2}>q_{1}$. We insert the two odd dots $e_{n+q_{1}}$ and $e_{n+q_{2}}$ in the $j$-th column of $T$. With precision, by the same argument as for $(a)$, those two dots are located between the lines $y=x$ and $y=x+n$. Afterwards, we set $\mathcal{I}_{i+1}^{o}:=\mathcal{I}_{i-1}^{o} \backslash\left\{q_{1}, q_{2}\right\}$. Thus, the sequence $\mathcal{I}_{i+1}^{o}$ has length $n-(i+2)+1$, and if $j \in\{i+2, i+3, \ldots, n\}$ then, by Hypothesis $H(i)$, the $(j-i-1)$-th element of $\mathcal{I}_{i-1}^{o}$ is inferior to $n+j-2$, so the $(j-(i+2)+1)$-th element of $\mathcal{I}_{i+1}^{o}$ is inferior to $n+j-2<n+j$. Therefore, Hypothesis $H(i+2)$ is true and we can iterate the algorithm to $i+2$.

2. Insertion of the $\boldsymbol{n}$ even $\operatorname{dots} \boldsymbol{e}_{\mathbf{1}}, \boldsymbol{e}_{\mathbf{2}}, \ldots, \boldsymbol{e}_{\boldsymbol{n}}$. Let $\mathcal{I}_{0}^{e}=(n, n-1, \ldots, 1)$. For $i=1$ to $n$, consider $j_{i} \in[n]$ such that the $(n+1-i)$-th up step $s_{n+1-i}^{u}$ of $\gamma$ is one of the 
two steps $\left(p_{2 j_{i}-2}, p_{2 j_{i}-1}\right)$ or $\left(p_{2 j_{i}-1}, p_{2 j_{i}}\right)$. If the sequence $\mathcal{I}_{i-1}^{e}$ is defined, we denote by $H^{\prime}(i)$ the hypothesis " $\mathcal{I}_{i-1}^{e}$ has length $n+1-i$ such that for all $j \in[n-i+1]$, the $(n-i+2-j)$-th element of $\mathcal{I}_{i-1}^{o}$ is greater than $j$ ". If Hypothesis $H^{\prime}(i+1)$ is true, we iterate the algorithm to $i+1$. In particular, the set $\mathcal{I}_{0}^{e}$ is defined and $H^{\prime}(1)$ is true so we can initiate the algorithm.

(a) If $s_{n+1-i}^{u}$ is an up step in the the context 1. or 2. of Definition 3.2, then let $i_{0} \in$ $[n]$ such that $\left\{\left(p_{2 j_{i}-2}, p_{2 j_{i}-1}\right),\left(p_{2 j_{i}-1}, p_{2 j_{i}}\right)\right\}=\left\{s_{n+1-i}^{u}, s_{i_{0}}^{d}\right\}$. Let $\left(n_{1}, n_{2}\right)=\xi_{i_{0}}$. From Remark 3.2, we have $1+n_{1} \leqslant 1+k=n-i+2-j_{i} \leqslant n-i+1$ so, following Hypothesis $H^{\prime}(i)$, we can consider the $\left(1+n_{1}\right)$-th element of $\mathcal{I}_{i-1}^{e}$, say, the integer $p$. We insert the even dot $e_{p}$ in the $j_{i}$-th column of $T$. By Hypothesis $H^{\prime}(i)$, the $\left(n-i+2-j_{i}\right)$-th element of $\mathcal{I}_{i-1}^{e}$ is greater than $j_{i}$, and $1+n_{1} \leqslant 1+k=n-i-j_{i}+2$ so the dot $e_{p}$ is located between the lines $y=x$ and $y=x+n$. Afterwards, we set $\mathcal{I}_{i}^{e}:=\mathcal{I}_{i-1}^{e} \backslash\{p\}$. The sequence $\mathcal{I}_{i}^{e}$ has length $n+1-(i+1)$. Also, if $j \in\{1,2, \ldots, n+1-(i+1)\}$, then, by Hypothesis $H^{\prime}(i)$, the $(n-i-j)$-th element of $\mathcal{I}_{i-1}^{e}$ is greater than $j+1$, so the $(n-(i+1)+1-j)$-th element of $\mathcal{I}_{i}^{e}$ is greater than $j+1>j$. Therefore, Hypothesis $H^{\prime}(i+1)$ is true and we can iterate the algorithm to $i+1$.

(b) If $s_{n+1-(i+1)}^{u}$ and $s_{n+1-i}^{u}$ are two consecutive up steps, say $\left(p_{2 j_{i}-2}, p_{2 j_{i}-1}\right)$ and $\left(p_{2 j_{i}-1}, p_{2 j_{i}}\right)$, from level $2 k-2$ towards level $2 k$ in $\gamma$, let $j_{0}>j_{i}$ such that the two steps $\left(p_{2 j_{0}-2}, p_{2 j_{0}-1}\right)$ and $\left(p_{2 j_{0}-1}, p_{2 j_{0}}\right)$ are the next two consecutive down steps $s_{i_{0}}^{d}$ and $s_{i_{0}+1}^{d}$ from level $2 k$ towards level $2 k-2$ (see Figure 9 ). Let $\left(n_{1}, n_{2}\right)=\xi_{i_{0}}$. Being in the context 3. of Definition 3.2, we have $n_{2} \leqslant n_{1} \leqslant$ $k-1=n-i-j_{0} \leqslant n-i-1$, hence $1+n_{2}<2+n_{1} \leqslant n-i+1$. Consequently, by Hypothesis $H^{\prime}(i)$, we can consider the $\left(1+n_{2}\right)$-th element of $\mathcal{I}_{i-1}^{e}$, say, the integer $p_{1}$, and the $\left(2+n_{1}\right)$-th element of $\mathcal{I}_{i-1}^{e}$, say, the integer $p_{2}<p_{1}$. We insert the two even dots $e_{p_{2}}$ and $e_{p_{1}}$ in the $j_{i}$-th column of $T$. With precision, for the same argument as for $(a)$, those two dots are between the lines $y=x$ and $y=x+n$. Afterwards, we set $\mathcal{I}_{i+1}^{e}:=\mathcal{I}_{i-1}^{e} \backslash\left\{p_{2}, p_{1}\right\}$. The sequence $\mathcal{I}_{i+1}^{e}$ has length $n-(i+2)+1$. Also, if $j \in\{1,2, \ldots, n+1-(i+2)\}$, then by Hypothesis $H^{\prime}(i)$, the $(n-i-j)$-th element of $\mathcal{I}_{i-1}^{e}$ is greater than $j+2$, so the $(n-(i+2)+2-j)$-th element of $\mathcal{I}_{i+1}^{e}$ is greater than $j+2>j$. Therefore, Hypothesis $H^{\prime}(i+2)$ is true and we can iterate the algorithm to $i+2$.

By construction, it is clear that $\Psi(S)=T$ is a Dellac configuration.

Remark 3.6. Let $S=(\gamma, \xi) \in D H(n)$ and $C=\Psi(S) \in D C(n)$. For all $i \in[n]$, the $i$-th up step $s_{i}^{u}$ (resp. down step $s_{i}^{d}$ ) of $\gamma$ gives birth to the even $\operatorname{dot} e_{p_{C}(i)}$ (resp. to the odd $\operatorname{dot} e_{n+q_{C}(i)}$ ) (see Definition 2.3).

Example 3.2. If $S \in D H(6)$ is the Dellac history $\Phi(C)$ of Example 3.1, we obtain $\Psi(S)=C$.

Following Remark 3.6, it is easy to prove the following lemma by induction on $i \in[n]$. 
Lemma 3.7. Let $S \in D H(n)$. We consider the two sequences $\left(\mathcal{I}_{i}^{o}\right)_{i}$ and $\left(\mathcal{I}_{i}^{e}\right)_{i}$ defined in the computation of $C=\Psi(S)$ (see Definition 3.6). Then for all $i \in[n]$, the integer $q_{C}(i)$ is the $\left(1+r_{C}^{o}\left(e_{n+q_{C}(i)}\right)\right)$-th element of the sequence $\mathcal{I}_{i-1}^{o}$, and the integer $p_{C}(n+1-i)$ is the $\left(1+l_{C}^{e}\left(e_{p_{C}(n+1-i)}\right)\right)$-th element of the sequence $\mathcal{I}_{i-1}^{e}$.

Proposition 3.8. The maps $\Phi: D C(n) \rightarrow D H(n)$ and $\Psi: D H(n) \rightarrow D C(n)$ are inverse maps.

Proof. From Remarks 3.4 and 3.6, it is easy to see that $\Phi \circ \Psi=I d_{D H(n)}$. The equality $\Psi \circ \Phi=I d_{D C(n)}$ is less straightforward. Let $C \in D C(n)$ and $S=(\gamma, \xi)=\Phi(C) \in D H(n)$. We are going to show, by induction on $i \in[n]$, that $q_{\Psi(S)}(i)=q_{C}(i)$ and $p_{\Psi(S)}(i)=p_{C}(i)$ for all $i$, hence $\Psi(S)=C$. The two proofs of $q_{\Psi(S)}(i)=q_{C}(i)$ and $p_{\Psi(S)}(i)=p_{C}(i)$ respectively being independant and analogous, we only prove $q_{\Psi(S)}(i)=q_{C}(i)$ for all $i$. Let $i=1$. In the context $1(a)$ of Definition 3.6, from Remark 3.4, the first odd dot to be inserted is $e_{n+q_{\Psi(S)}(1)}$. Therefore, by definition, the integer $q_{\Psi(S)}(1)$ is the $\left(1+n_{2}\right)$-th element of $\mathcal{I}_{0}^{o}$ (i.e., we obtain $q_{\Psi(S)}(1)=1+n_{2}$ where $\left.\left(n_{1}, n_{2}\right)=\xi_{1}\right)$. In this situation, since $S=\Phi(C)$, we know that $n_{2}=r_{C}^{o}\left(e_{n+q_{C}(1)}\right)$. Consequently, from Lemma 3.7, we obtain $q_{\Psi(S)}(1)=1+r_{C}^{o}\left(e_{n+q_{C}(1)}\right)=q_{C}(1)$. The proof in the context $1(b)$ is analogous. Now let $i \in\{2,3, \ldots, n\}$. Suppose that $q_{\Psi(S)}(k)=q_{C}(k)$ for all $k<i$. In the context $1(a)$ of Definition 3.6, from Remark 3.4, the $i$-th odd dot to be inserted is $e_{n+q_{\Psi(S)}(i)}$. Therefore, by definition, if $\xi_{i}=\left(n_{1}, n_{2}\right)$, then $q_{\Psi(S)}$ is the $\left(1+n_{2}\right)$-th element of $\mathcal{I}_{i-1}^{e}=\mathcal{J}_{i-1}^{e}$. Since $S=\Phi(C)$, we know that $n_{2}=r_{C}^{o}\left(e_{n+q_{C}(i)}\right)$ so, from Lemma 3.7, we obtain $q_{\Psi(S)}(i)=q_{C}(i)$. The proof in the context $1(b)$ is analogous.

This proves Theorem 3.3.

As an illustration of the whole paper, the table depicted in the next page makes explicit the statistic-preserving bijections between the $h_{3}=7$ objects of $D C(3), \mathcal{D}_{4}^{\prime}$ and $D H(3)$.

\section{Acknowledgements}

I thank Jiang Zeng for his comments and useful references. 


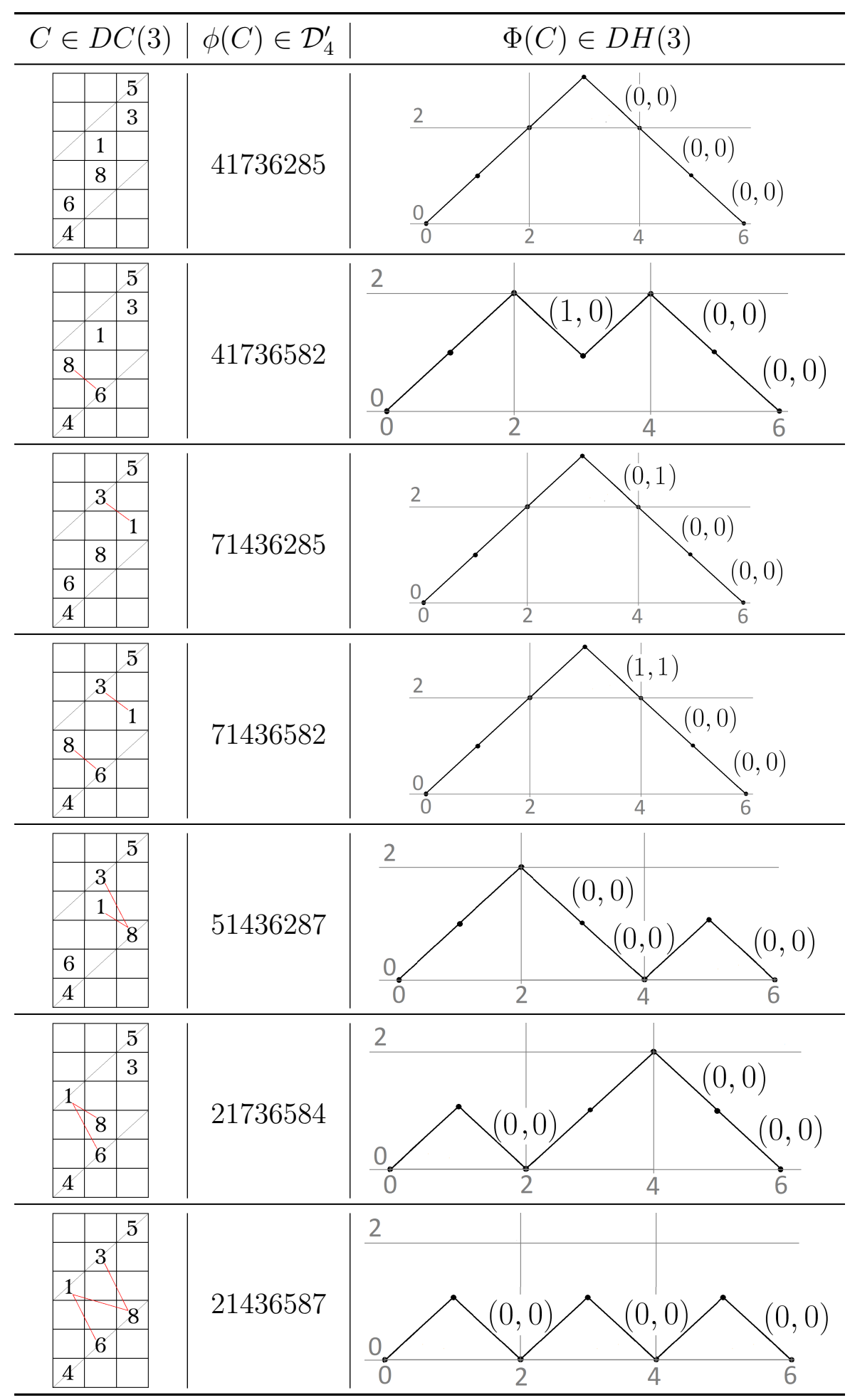




\section{References}

[1] D. Barsky and D. Dumont. Congruences pour les nombres de Genocchi de 2e espèce. (French) Study Group on Ultrametric Analysis. 7th-8th years: 1979-1981 (Paris, 1979/1981) (French), Exp. No. 34, 13 pp., Secrétariat Math., Paris, 1981.

[2] H. Dellac. Problem 1735. L'Intermédiaire des Mathématiciens 7:9-10, 1900.

[3] D. Dumont. Interprétations combinatoires des nombres de Genocchi. (French) Duke Math. J. 41 (1974), 305-318.

[4] D. Dumont and A. Randrianarivony. Dérangements et nombres de Genocchi. (French. English summary) [Derangements and Genocchi numbers] Discrete Math. 132 (1994), no. $1-3,37-49$.

[5] D. Dumont and G. Viennot. A combinatorial interpretation of the Seidel generation of Genocchi numbers. Ann. Discrete Math. 6 (1980), 77-87.

[6] E. Feigin. Degenerate flag varieties and the median Genocchi numbers. Math. Res. Lett. 18 (2011), no. 6, 1163-1178.

[7] E. Feigin. The median Genocchi numbers, q-analogues and continued fractions. European J. Combin. 33 (2012), no. 8, 1913-1918.

[8] P. Flajolet. Combinatorial aspects of continued fractions. Discrete Math. 32 (1980), no. $2,125-161$.

[9] G.-N. Han and J. Zeng. On a $q$-sequence that generalizes the median Genocchi numbers. Ann. Sci. Math. Qubec 23 (1999), no. 1, 63-72.

[10] G.-N. Han and J. Zeng. q-polynômes de Gandhi et statistique de Denert. (French. English summary) [Gandhi q-polynomials and Denert statistics] Discrete Math. 205 (1999), no. 1-3, 119-143.

[11] G. Cerulli Irelli and E. Feigin and M. Reineke. Quiver Grassmannians and degenerate flag varieties. Algebra \& Number Theory 6 (2012), no. 1, 165-194.

[12] G. Kreweras. Sur les permutations comptées par les nombres de Genocchi de première et deuxième espèce. (French) [Permutations enumerated by Genocchi numbers of the first and the second kind] European J. Combin. 18 (1997), no. 1, 49-58.

[13] OEIS Foundation Inc. (2011). The On-Line Encyclopedia of Integer Sequences. http://oeis.org/A110501

[14] OEIS Foundation Inc. (2011). The On-Line Encyclopedia of Integer Sequences. http://oeis.org/A005439

[15] OEIS Foundation Inc. (2011). The On-Line Encyclopedia of Integer Sequences. http://oeis.org/A000366

[16] G. Viennot. Interprétations combinatoires des nombres d'Euler et de Genocchi. (French) [Combinatorial interpretations of Euler and Genocchi numbers] Seminar on Number Theory, 1981/1982, Exp. No. 11, 94 pp., Univ. Bordeaux I, Talence, 1982. 